Document downloaded from:

http://hdl.handle.net/10251/100415

This paper must be cited as:

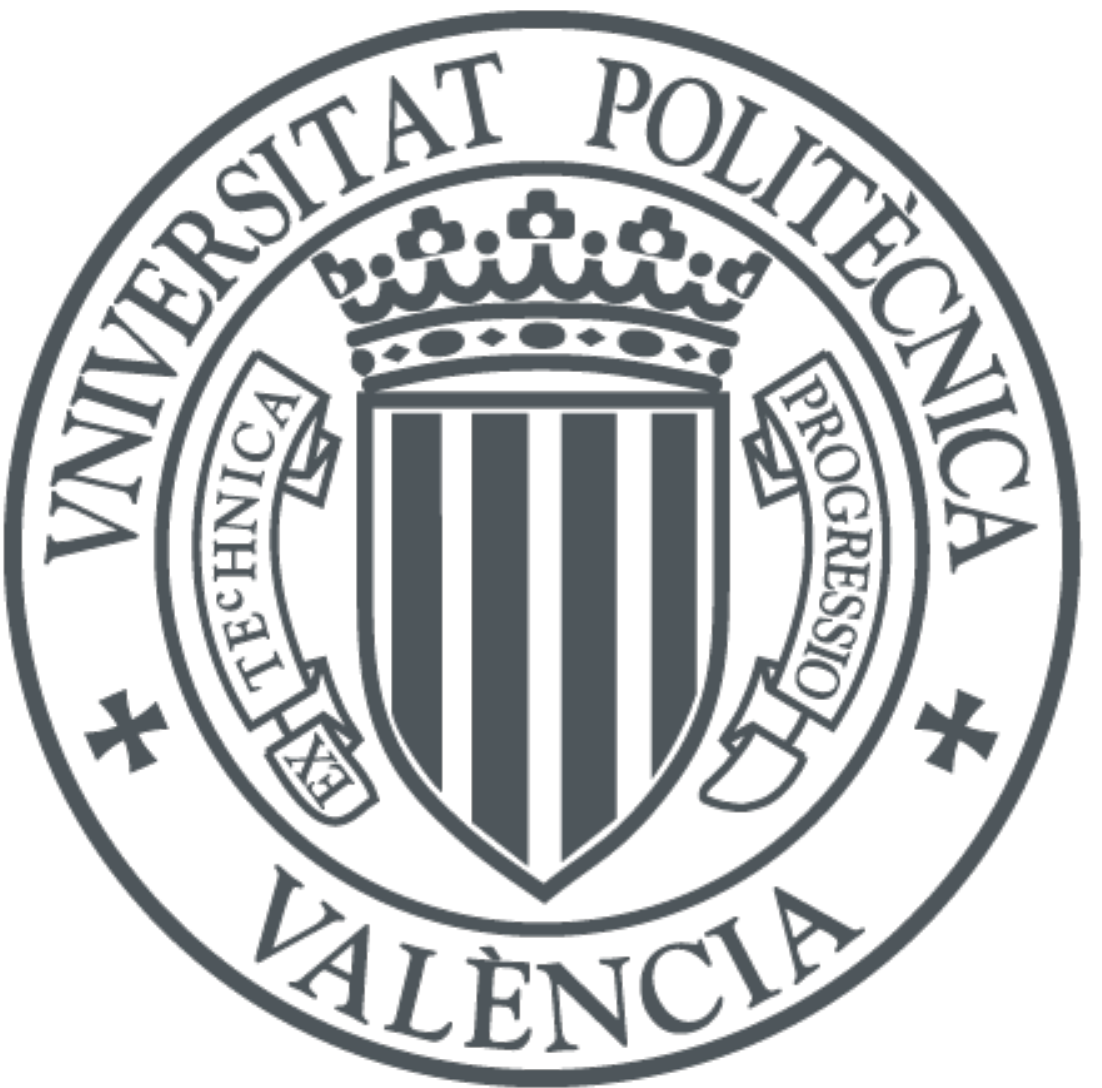

The final publication is available at

http://doi.org/10.1007/s10107-003-0391-9

Copyright Springer-Verlag

Additional Information 


\title{
The Mixed General Routing Polyhedron
}

\author{
A. Corberán ${ }^{1 *}$, A. Romero ${ }^{1}$ and J.M. Sanchis ${ }^{2}$ \\ ${ }^{1}$ Dept. d'Estadística i Investigació Operativa, Universitat de València, Spain \\ 2 Dept. de Matemática Aplicada, Universidad Politécnica de Valencia, Spain
}

April 1999

First revision: May 2000

Second revision: May 2001

\begin{abstract}
In Arc Routing Problems, ARPs, the aim is to find on a graph a minimum cost traversal satisfying some conditions related to the links of the graph. Due to restrictions to traverse some streets in a specified way, most applications of ARPs must be modeled with a mixed graph. Although several exact algorithms have been proposed, no polyhedral investigations have been done for ARPs on a mixed graph. In this paper we deal with the Mixed General Routing Problem which consists of finding a minimum cost traversal of a given link subset and a given vertex subset of a mixed graph. A formulation is given that uses only one variable for each link (edge or arc) of the graph. Some properties of the associated polyhedron and some large families of facet-inducing inequalities are described. A preliminary cutting-plane algorithm has produced very good lower bounds over a set of 100 randomly generated instances of the Mixed Rural Postman Problem. Finally, applications of this study to other known routing problems are described.
\end{abstract}

Key Words: Polyhedral Combinatorics, Facets, Routing, Arc Routing, Rural Postman Problem, General Routing Problem, Mixed Chinese Postman Problem.

\section{Introduction}

Arc Routing Problems, ARPs, have their origin in the celebrated Königsberg Bridge Problem solved by Euler and consist, basically, of finding a set of routes over the arcs (or edges) of a graph satisfying certain restrictions. There are many real situations in which such a set of routes is required: collection or delivery of goods, mail distribution, network maintenance (electrical lines or gas mains inspection), school

*corresponding author: angel.corberan@uv.es 
bus transportation, garbage collection, etc. For this reason, a big number of Arc Routing Problems have been studied in the last 30 years. Papers by Eiselt, Gendreau \& Laporte (1995a,1995b), by Assad \& Golden (1995) and the recent book edited by Dror (2000) survey the state of the art and real-life applications of the ARPs. In most applications, there are some restrictions for the vehicle to traverse some streets in a specified way, and the problem needs to be modeled with a mixed graph. As a rule, one-way streets are represented by arcs and two-way streets by edges. We will use the word link to refer to both an edge or an arc.

In this paper we study the General Routing Problem defined on a mixed graph, the Mixed General Routing Problem (MGRP), that we define next:

Given a strongly connected mixed graph $G=(V, E, A)$ with vertex set $V$, edge set $E$, arc set $A$, a cost $c_{e}$ for each $e \in E \cup A$, a set $E_{R} \subseteq E$ of required edges, a set $A_{R} \subseteq A$ of required arcs and a set $V_{R} \subseteq V$ of required vertices, the Mixed General Routing Problem (MGRP) is the problem of finding a minimum cost vehicle route passing through each $e \in E_{R} \cup A_{R}$ and through each $i \in V_{R}$ at least once.

The MGRP has several well known routing problems as special cases. Given an undirected and connected graph $\mathrm{G}=(\mathrm{V}, \mathrm{E})$ with nonnegative costs associated with its edges, the Chinese Postman Problem (CPP) consists of finding a minimum cost tour (a closed walk) traversing, at least once, all the edges in E. This well known problem is solvable in polynomial time (Edmonds \& Johnson, 1973). However, if the tour has to traverse only a subset, $E_{R}$, of the set of edges $E$ and this subset induces in $G$ a disconnected graph, the problem, called the Rural Postman Problem (RPP), turns out to be NP-hard (Lenstra \& Rinnooy-Kan, 1976). Furthermore, if the condition for the tour is to traverse both a given subset of edges $E_{R} \subset E$ and a given subset of vertices $V_{R} \subset V$, the problem is then called the General Routing Problem (GRP) and it is also NP-hard. On the other hand, if the CPP is defined over a mixed graph, the problem of traversing all the arcs and edges in $\mathrm{G}$ (the Mixed Chinese Postman Problem, MCPP) is again NP-hard (Papadimitriou, 1976).

Although the MGRP is not strictly an ARP, it is a generalization of both the Mixed RPP (MRPP) and the Graphical Asymmetric TSP (GATSP). The MRPP contains the (undirected) Rural Postman Problem and the Mixed Chinese Postman Problem as particular cases and, thus, is a NP-hard problem. The Graphical Asymmetric TSP consists of finding a minimum cost tour on a strongly connected directed graph $G=(V, A)$ traversing each vertex of $G$ at least once. This problem, recently studied by Chopra and Rinaldi (1996), is clearly a special case of MGRP when $E=\emptyset$, all the arcs are non-required and all the vertices are required.

The existing optimal approaches for ARP's on mixed graphs are related to the MCPP. Christofides et al. (1984) present a formulation for the MCPP in which a variable is associated with each arc, two variables with each edge (representing the number of times the edge is traversed in each direction) and another variable with each 
vertex. Then, a branch and bound algorithm was implemented that solved 34 small size instances. Grötschel \& Win (1992) propose a cutting-plane algorithm to solve the Windy Postman Problem (WPP), the problem of finding a minimum cost tour traversing all edges of an undirected graph with two costs associated with each edge (the cost of traversing an edge depends on the direction of travel). The WPP, that contains the MCPP as a special case, is also formulated using two variables associated with each edge. Nine medium size MCPP instances were solved to optimality with this cutting-plane algorithm. The formulations in both papers are based on transforming the original graph into a directed graph that is eulerian if symmetry conditions on the vertices of the graph are satisfied. A somewhat different approach that uses only one variable associated with each edge was proposed by Nobert \& Picard (1996). It is based on the characterization of an eulerian mixed graph given by Ford \& Fulkerson (1962): a strongly connected mixed graph is eulerian if and only if the degree of each node is even (evenness condition) and for every proper subset of vertices $S$, the number of arcs entering $S$ minus the number of arcs leaving $S$ is less than or equal to the number of edges between $S$ and $V \backslash S$ (balanced sets condition). Nobert \& Picard present a cutting-plane algorithm that was able to solve 148 instances out of 180 randomly generated instances with sizes up to 169 vertices, 2876 arcs and 1849 edges.

Since there is reasonably strong evidence that the last approach is computationally superior to the previous one, we chose to formulate the MGRP in this way. In Section 2 we present the formulation of the problem and in Section 3 we study its associated polyhedron. Some basic inequalities that are valid for the polyhedron and that, under certain conditions, define facets of it, are presented in Section 4: trivial, connectivity, balanced-set and $R$-odd cut constraints. In Sections 5 and 6 , two big families of inequalities, Path Bridge and Path Bridge $0_{2}$ inequalities, are presented and shown to be facet inducing of the MGRP polyhedron. Some computational results are presented in Section 7, where a preliminary cutting-plane algorithm, based on a part of the given description of the MGRP polyhedron, has produced very good lower bounds over a set of 100 randomly generated instances of the MRPP. Finally, in Section 8 we apply the results from this study to other known Routing Problems.

\section{Problem definition and notation}

Note that if $i \in V$ is a vertex incident to any required link $e \in E_{R} \cup A_{R}$, the condition on the tour passing through edge $e$ contains the condition of visiting vertex $i$. Therefore, in the following, we will assume that $V_{R}$ contains the set of vertices incident to the required edges.

As it is usual when the subgraph induced by the required vertices and links is not connected, we first transform the original graph in order to simplify both the problem structure and the formulation. This transformation is done in a similar way to that of Christofides et al. (1981) for the undirected RPP (see Eiselt, Gendreau \& Laporte, 1995b, or Christofides et al., 1986, for an illustration of the procedure): 
1. Add to $G_{R}=\left(V_{R}, E_{R} \cup A_{R}\right)$ an arc between every pair of vertices in $V_{R}$ having a cost equal to the shortest path length on $G$ if such a path does not uses required links.

2. Delete: a) one of every two arcs in parallel if they have the same cost, b) every arc parallel to an edge with the same cost, and c) all $\operatorname{arcs} e=(i, j) \notin E_{R}$ such that $c_{i j}=c_{i k}+c_{k j}$ for some vertex $k$.

This transformation can occasionally increase the number of edges in $G$, but it frequently decreases it. Notice that all vertices in the simplified graph are either required or incident to a required link and that $E \backslash E_{R}=\emptyset$. Hence, we can assume that we work with a (simplified) strongly connected graph $G=(V, E, A):=\left(V_{R}, E_{R}, A_{R} \cup A_{N R}\right)$.

Let $G^{R}=G\left(V, E, A_{R}\right)$ be the graph obtained by deleting in $G$ all the non required arcs. In general, graph $G^{R}$ is not connected. Let $p$ be the number of connected components of $G^{R}$ and let $V_{1}, V_{2}, \ldots, V_{p}$ be the vertex sets corresponding to the $p$ connected components of $G^{R}$, which will be called $R$-sets, with $V_{1} \cup \ldots \cup V_{p}=V$. We will represent by $C_{i}=G\left(V_{i}\right), i=1, \ldots, p$, the subgraphs of $G$ induced by the $R$-sets and they will be referred to as $R$-connected components. Notice that every isolated required vertex is a R-connected component of $G$. Given two disjoint sets of vertices $S_{1}, S_{2} \subset V$ and a set $S \subset V$, we will use the following notation:

$$
\begin{aligned}
& \left(S_{1}: S_{2}\right)=\left\{(i, j) \in E \cup A: i \in S_{1}, j \in S_{2} \quad \text { or } \quad i \in S_{2}, j \in S_{1}\right\} \\
& A\left(S_{1}: S_{2}\right)=\left\{(i, j) \in A: i \in S_{1}, j \in S_{2}\right\} \\
& E\left(S_{1}: S_{2}\right)=\left(S_{1}: S_{2}\right) \cap E \\
& \delta(S)=(S: V \backslash S)(\text { called link cut-set of } G \text { defined by } S) \\
& \gamma(S)=\{(i, j) \in E \cup A: i, j \in S\} \\
& A^{+}(S)=A(S: V \backslash S) \\
& A^{-}(S)=A(V \backslash S: S) \\
& A(S)=A^{+}(S) \cup A^{-}(S) \\
& E(S)=E(S: V \backslash S)
\end{aligned}
$$

The above sets are defined in a similar way referring to required links only and to non-required links only: $\left(S_{1}: S_{2}\right)_{R}, A_{N R}\left(S_{1}: S_{2}\right), A_{R}^{+}(S), A_{N R}^{-}(S), \delta_{R}(S)$, etc. Given $x \in \mathbb{R}^{E \cup A}$ and given $T \subset E \cup A, x(T)$ denotes $\sum_{e \in T} x_{e}$.

A tour for the $M G R P$ is a family $\mathcal{F}$ of links of $G$ such that the graph $\left(V, E^{\mathcal{F}} \cup A^{\mathcal{F}}\right)$ is unicursal (also called eulerian) and contains all the required links, where $E^{\mathcal{F}} \cup A^{\mathcal{F}}$ is obtained by considering each copy of a link in $\mathcal{F}$ as a different element. Applying sufficient conditions for a connected mixed graph to be unicursal (Ford and Fulkerson, 1962), we can state that $\mathcal{F}$ is a tour for the MGRP when the following conditions are satisfied:

- $\mathcal{F}$ contains all the required links.

- Graph $\left(V, E^{\mathcal{F}} \cup A^{\mathcal{F}}\right)$ is even, i.e., every vertex is incident to an even number of links. 
- $\operatorname{Graph}\left(V, E^{\mathcal{F}} \cup A^{\mathcal{F}}\right)$ is connected.

- Graph $\left(V, E^{\mathcal{F}} \cup A^{\mathcal{F}}\right)$ is balanced, i.e., for every $S \subset V$, the difference between the number of arcs in $A^{+}(S)$ (leaving $S$ ) and the number of arcs in $A^{-}(S)$ (entering $S$ ) is less than or equal to the number of edges in $E(S)$ (balanced-set condition for set $S$ ).

We formulate the MGRP with respect to semitours. We call the family of links obtained from any tour for the MGRP by deleting one copy of every link in $E \cup A_{R}$ a semitour for the MGRP. We associate with each tour (semitour) an integer incidence vector $x=\left(x_{e}: \quad e \in E \cup A\right) \in \mathbb{R}^{E \cup A}$, where $x_{e}$ denotes the number of times a link $e \in E \cup A$ appears in the tour (semitour). For notational convenience, we will use also the word tour (semitour) to denote this vector. Let $x^{R}$ denotes the incidence vector of required links. Then, $x$ is a semitour for the MGRP on $G$ if, and only if, $x+x^{R}$ is a tour. Vector $x+x^{R}$ will be referred to as the corresponding tour of a given semitour $x$. A vertex $v \in V$ will be called $R$-odd if it is incident to an odd number of required links, otherwise it will be called $R$-even. Note that every isolated required vertex is R-even. Given $S \subset V$, let $u_{S}=\left|A_{R}^{+}(S)\right|-\left|A_{R}^{-}(S)\right|-|E(S)|$. It is easy to see that the set of semitours for the MGRP is the set of vectors $x \in \mathbb{R}^{E \cup A}$ satisfying:

$$
\begin{aligned}
x_{e} \geq 0 \text { and integer, } & \forall e \in E \cup A \\
x(\delta(\{i\})) \equiv 0 \bmod 2, & \forall i \in V: v \text { is } R \text { - even } \\
x(\delta(\{i\})) \equiv 1 \bmod 2, & \forall i \in V: v \text { is } R-\text { odd } \\
x\left(A^{+}(S)\right) \geq 1, & \forall S=\cup_{k \in Q} V_{k}, Q \subset\{1, \ldots, p\} \\
-x\left(A^{+}(S)\right)+x\left(A^{-}(S)\right)+x(E(S)) \geq u_{S}, & \forall S \subset V
\end{aligned}
$$

where conditions (2) and (3), (4) and (5) force the graph represented by the tour to be, respectively, even, connected and balanced.

Let us describe briefly constraints (5) (see figure 1, where the required links are represented in solid lines and the non required in dash lines). Given that $u_{S}=1$, constraint (5) can be expressed as $x\left(A^{-}(S)\right)+x(E(S)) \geq 1+x\left(A^{+}(S)\right)$. It could be understood that we must add, at least, $u_{S}=1$ link in the set $A^{-}(S) \cup E(S)$ in order to obtain a balanced graph. If we consider now $\bar{S}=V \backslash S$, then $u_{\bar{S}}=\left|A_{R}^{-}(S)\right|-\left|A_{R}^{+}(S)\right|-|E(S)|=-3$ and the corresponding constraint (5) can be expressed as $x\left(A^{-}(S)\right) \leq 3+x\left(A^{+}(S)\right)+x(E(S))$. It means that we can not add more than 3 links in $A^{-}(S)$, if we do not add also links in $A^{+}(S) \cup E(S)$.

Consider now a set $S \subset V$ such that $E(S)=\emptyset$. Then $u_{S}=-u_{\bar{S}}$ and constraints (5) corresponding to $S$ and $\bar{S}$ imply the so called symmetry equation $x\left(A^{+}(S)\right)+u_{S}=$ $x\left(A^{-}(S)\right)$. Hence, system (1) to (5) includes an equation associated with each set $S \subset V$ with $E(S)=\emptyset$, most of them linearly dependent. In order to discuss this, let us represent by $K_{1}, K_{2}, \ldots, K_{q}$ the sets of vertices of the connected components of the graph $(V, E)$. Some sets $K_{i}$ could consist in a single vertex. Each set $K_{i}$ is contained 


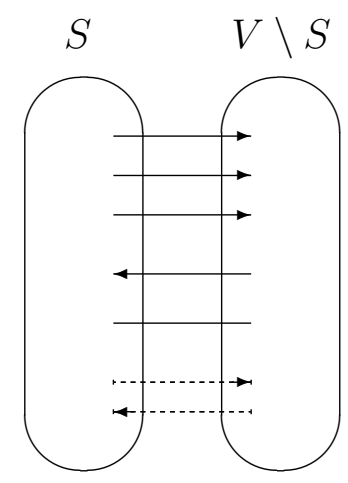

$$
\begin{aligned}
& u_{S}=1: \quad x\left(A^{-}(S)\right)+x(E(S)) \geq 1+x\left(A^{+}(S)\right) \\
& u_{\bar{S}}=-3: \quad x\left(A^{-}(S)\right) \leq 3+x\left(A^{+}(S)\right)+x(E(S))
\end{aligned}
$$

Figure 1: Some examples of balanced set constraints.

in a set $V_{j}$. We will call the $q$ subgraphs of $G$ induced by sets $K_{i}$ edge-connected components of $G$. The $q$ equations

$$
x\left(A^{+}\left(K_{i}\right)\right)+u_{K_{i}}=x\left(A^{-}\left(K_{i}\right)\right), \quad i=1,2, \ldots, q
$$

will be referred as the system equations. It can be shown that any $q-1$ of them are linearly independent.

\section{Basic Properties}

Let $\operatorname{MGRP}(G)$ be the convex hull in $\mathbb{R}^{E \cup A}$ of all the semitours for the MGRP on $G$, i.e., of all vectors $x \in \mathbb{R}^{E \cup A}$ satisfying (1) to (5). It is not hard to see that $\operatorname{MGRP}(G)$ is an unbounded polyhedron. In order to study the dimension of $\operatorname{MGRP}(G)$, we will use the following result, due to Chopra \& Rinaldi (1996). Let $\operatorname{GATSP}(G)$ be the convex hull of the tours for the GATSP on a directed graph $G$. Then,

Lemma 1 (Chopra \& Rinaldi, 1996) Let $G=(V, A)$ be a directed graph. If $G$ is strongly connected, $\operatorname{dim}(G A T S P(G))=|A|-|V|+1$

Theorem $1 \operatorname{dim}(M G R P(G))=|E \cup A|-q+1$, where $q$ is the number of edge-connected components of $G$.

Proof: As every semitour satisfies equations (6) and $q-1$ of them are linearly independent, $\operatorname{dim}(\operatorname{MGRP}(G)) \leq|E \cup A|-q+1$ holds. In order to prove the equality, we will find $|E \cup A|-q+2$ affinely independent semitours for the MGRP on $G$.

Let $F=A \backslash\left(\gamma\left(K_{1}\right) \cup \ldots \cup \gamma\left(K_{q}\right)\right)$ be the set of arcs joining vertices from two different sets $K_{i}, K_{j}$. Let $G_{s}$ be the graph obtained by shrinking node sets $K_{i}, i=1, \ldots, q$ into a single vertex each. Graph $G_{s}$ is a strongly connected directed graph with $q$ vertices and with an arc corresponding to each arc in $F$. Note that $G_{s}$ can have parallel arcs. From lemma $1, \operatorname{dim}\left(\operatorname{GATSP}\left(G_{s}\right)\right)=|F|-q+1:=m$ and hence we can find $m+1$ affinely independent tours for the GATSP on $G_{s}$. If we add to these tours the incidence 
vector of a cycle on $G_{s}$ traversing all the arcs corresponding to the required arcs in $F$, we obtain $m+1$ new tours for the GATSP on $G_{s}$ that are also affinely independent. Now, it is easy to transform these tours by adding edges and arcs in $\gamma\left(K_{1}\right) \cup \ldots \cup \gamma\left(K_{q}\right)$ in order to obtain $m+1$ tours for the MGRP on $G$. Let $x^{0}, x^{1}, \ldots, x^{m}$ denote their corresponding $m+1$ semitours.

For each edge $e \in \gamma\left(K_{1}\right) \cup \ldots \cup \gamma\left(K_{q}\right)$, consider now the semitour obtained by adding to $x^{0}$ (for instance) two copies of $e$. For each arc $a \in \gamma\left(K_{i}\right), i=1, \ldots, q$, consider the semitour obtained by adding to $x^{0}$ a copy of $a$ and a copy of each edge in a path in $G\left(K_{i}\right)$ using only edges (such a path exists because of the definition of $K_{i}$ ). Then we have $\left|\gamma\left(K_{1}\right) \cup \ldots \cup \gamma\left(K_{q}\right)\right|$ more semitours for the MGRP on $G$.

Consider the matrix obtained by expressing the semitours as rows and the links as columns. Subtracting the row corresponding to $x^{0}$ from the remaining rows, we obtain the matrix in figure 2, where $I$ is the identity matrix and $B$ is the matrix, with rank $m$, corresponding to the tours for the GATSP. Then, the rank of the full matrix is $\left|\cup \gamma\left(K_{i}\right)\right|+m=\left|\cup \gamma\left(K_{i}\right)\right|+|F|-q+1=|E \cup A|-q+1$ and we have found $|E \cup A|-q+2$ affinely independent semitours for the MGRP on $G$.

\begin{tabular}{|c|c|c|}
\hline$\left(\cup \gamma\left(K_{i}\right)\right) \cap A$ & $\left(\cup \gamma\left(K_{i}\right)\right) \cap E$ & $F$ \\
\hline $\mathrm{I}$ & $*$ & $*$ \\
\hline 0 & $2 \mathrm{I}$ & $*$ \\
\hline 0 & 0 & $\mathrm{~B}$ \\
\hline
\end{tabular}

Figure 2: Matrix appearing in the proof of theorem 1

Given that $\operatorname{MGRP}(G)$ is not full-dimensional, different inequalities can induce the same facet. Such inequalities are called equivalent. We next study conditions for inequalities (1) without the integrality condition, which will be called trivial inequalities, to be facet-inducing. A link $e \in E \cup A$ will be called a cut-link of $G$ if, after removing $e$ from $G$, the resulting graph is not strongly connected.

Theorem 2 (Trivial inequalities) : Inequalities $x_{e} \geq 0, \forall e \in E \cup A$, are facetinducing for $M G R P(G)$ if $e$ is not a cut-link of $G$ (the condition is also necessary for links e $\left.\in E \cup A_{N R}\right)$.

Proof: The proof is simple and is omitted here for the sake of brevity. 
Before studying the remaining inequalities in the formulation, we describe some general results that will be useful in what follows.

Let $G=(V, E, A)$ be a mixed graph. A configuration $\mathcal{C}$ on $G$ is a $\operatorname{triplet}\left(\mathcal{B}, c^{E}, c^{A}\right)$, where $\mathcal{B}=\left\{B_{1}, B_{2}, \ldots, B_{r}\right\}$ is a partition of $V, c^{E}$ and $c^{A}$ are real functions defined on $\mathcal{B} \times \mathcal{B}$ and the following conditions are satisfied:

$\left(\mathcal{C}\right.$-a) Every subgraph $G\left(B_{i}\right)$ is strongly connected.

$\left(\mathcal{C}\right.$-b) $c^{E}\left(B_{i}, B_{j}\right)=c^{E}\left(B_{j}, B_{i}\right)>0$ for every pair $B_{i} \neq B_{j}$ such that $E\left(B_{i}: B_{j}\right) \neq \emptyset$

$\left(\mathcal{C}\right.$-c) There is no closed cycle $B_{i}, B_{j}, \ldots, B_{m}, B_{i}$ with total $c$-cost negative.

Associated with a configuration $\mathcal{C}$ on $G$ we have a configuration graph $G_{\mathcal{C}}$ having node set $\mathcal{B}$, a required edge $\left(B_{i}, B_{j}\right)$ for each required edge $(u, v)$ of $G$ with $u \in B_{i}$, $v \in B_{j}$, a required arc $\left(B_{i}, B_{j}\right)$ for each required $\operatorname{arc}(u, v)$ of $G$ with $u \in B_{i}, v \in B_{j}$ and a non required arc $\left(B_{i}, B_{j}\right)$ for each pair $B_{i}, B_{j}$ such that $A_{N R}\left(B_{i}: B_{j}\right) \neq \emptyset$. In other words, $G_{\mathcal{C}}$ is the graph resulting from shrinking node sets $B_{i}, i=1, \ldots, r$ into a single vertex each and, after that, shrinking each set of non required parallel arcs into one single arc, but keeping all the required edges and arcs. A configuration $\mathcal{C}$ defines a configuration inequality, $\sum_{e \in E \cup A} c_{e} x_{e} \geq c_{0}$, where:

(a) $c_{e}=0$ for every $e \in \gamma\left(B_{i}\right), i=1, \ldots, r$

(b) $c_{e}=c^{E}\left(B_{i}, B_{j}\right)$ for every edge $e \in E\left(B_{i}: B_{j}\right)$

(c) $c_{a}=c^{A}\left(B_{i}, B_{j}\right)$ for every arc $a \in A\left(B_{i}: B_{j}\right)$

(d) $c_{0}$ is the $c$-length of the shortest semitour for the MGRP on $G_{\mathcal{C}}$ (and on $G$ ).

Notice that it is possible to have $c^{A}\left(B_{i}, B_{j}\right) \neq c^{A}\left(B_{j}, B_{i}\right) \neq c^{E}\left(B_{i}, B_{j}\right)$ and also $c^{A}\left(B_{i}, B_{j}\right)<0$ (see connectivity and balanced-set inequalities (4) and (5)).

Theorem 3 All facet-inducing inequalities for $M G R P(G)$, except those equivalent to trivial ones, are configuration inequalities.

Proof: The proof is similar to that in Naddef and Rinaldi (1991) for the Graphical TSP, and is omitted here for the sake of brevity.

The following results are two 'lifting' theorems that state conditions for a given configuration inequality which is facet-inducing for $\operatorname{MGRP}\left(G_{\mathcal{C}}\right)$ to be also facet-inducing for $\operatorname{MGRP}(G)$. Given a mixed graph $G=(V, E, A), q(G)$ will denote the number of edge-connected components of $G$, i.e., the number of connected components of the graph $(V, E)$.

Theorem 4 Let $G$ be a mixed graph and let $\mathcal{C}$ be a configuration on $G$. The associated configuration inequality is facet-inducing for $M G R P(G)$ if the configuration inequality associated with $\mathcal{C}$ on graph $G_{\mathcal{C}}$ is facet-inducing for $M G R P\left(G_{\mathcal{C}}\right)$ and the following condition is satisfied:

$$
q(G)=q\left(G_{\mathcal{C}}\right)+\sum_{i=1}^{r}\left(q\left(G\left(B_{i}\right)\right)-1\right)
$$


Proof: By induction, it suffices to prove the result for a graph $G$ obtained from $G_{\mathcal{C}}$ by replacing node $B_{1}$ (for example) by a strongly connected graph $G\left(B_{1}\right)$. For simplicity, $F(x) \geq c_{0}$ will denote both the inequality on $G$ and the inequality on $G_{\mathcal{C}}$. We first show validity. Consider any semitour $x$ for the MGRP on $G$. Shrinking $G\left(B_{1}\right)$ into a single vertex we obtain a semitour $x^{\prime}$ for the MGRP on $G_{\mathcal{C}}$ satisfying $F(x)=F\left(x^{\prime}\right) \geq c_{0}$.

Let $K=\operatorname{dim}\left(\operatorname{MGRP}\left(G_{\mathcal{C}}\right)\right)$ and let us suppose that $c_{0} \neq 0$. Then, there exists $K$ linearly independent semitours for the MGRP on $G_{\mathcal{C}}$ satisfying $F(x)=c_{0}$, say $x^{1}, x^{2}, \ldots, x^{K}$. If $c_{0}=0$ there would be only $K-1$ semitours, but the proof would be similar. After replacing $B_{1}$ by the graph $G\left(B_{1}\right)$, some non required arcs may appear between the vertices of $G\left(B_{1}\right)$ and nodes $B_{i}, i \neq 1$. Let us call $G_{\mathcal{C}}^{\prime}$ the graph obtained from $G_{\mathcal{C}}$ by adding $Q$ non required $\operatorname{arcs} e_{1}^{\prime}, e_{2}^{\prime}, \ldots, e_{Q}^{\prime}$, where each $e_{i}^{\prime}$ is parallel to a non required arc (not necessarily distinct) $e_{i}, i=1,2, \ldots, Q$, of $G_{\mathcal{C}}$. For each $e_{i}^{\prime}$, let $x^{j}$ be the semitour for the MGRP on $G_{\mathcal{C}}$ traversing $e_{i}$ and consider the semitour $x^{j} \backslash\left\{e_{i}\right\} \cup\left\{e_{i}^{\prime}\right\}$. Then, we have $K+Q$ linearly independent semitours for the MGRP on $G_{\mathcal{C}}^{\prime}$ satisfying $F(x)=c_{0}$. Given that $G\left(B_{1}\right)$ is strongly connected, each of them can be completed with links of $G\left(B_{1}\right)$ in order to obtain semitours for the MGRP on $G$ also satisfying $F(x)=c_{0}$. Let us call them $x^{1}, x^{2}, \ldots, x^{K+Q}$.

Consider the MGRP defined on graph $G\left(B_{1}\right)$, let $M=\operatorname{dim}\left(\operatorname{MGRP}\left(G\left(B_{1}\right)\right)\right)$ and let $y^{1}, y^{2}, \ldots, y^{M}$ be $M$ linearly independent semitours for the MGRP on $G\left(B_{1}\right)$. Then, $x^{1}+y^{1}, x^{1}+y^{2}, \ldots, x^{1}+y^{M}$ are also semitours for the MGRP on $G$ satisfying $F(x)=c_{0}$. Expressing the $K+Q+M$ semitours as rows of a matrix and subtracting the first row $x^{1}$ from the last $M$ rows, we obtain a full rank matrix. Hence, we have found $K+Q+M$ linearly independent semitours for the MGRP on $G$ satisfying $F(x)=c_{0}$. The proof will be completed when $\operatorname{dim}(\operatorname{MGRP}(G))=K+Q+M$ is showed.

Given that graphs $G_{\mathcal{C}}$ and $G_{\mathcal{C}}^{\prime}$ have the same set of edges, it holds that $q\left(G_{\mathcal{C}}\right)=$ $q\left(G_{\mathcal{C}}^{\prime}\right)$ and condition (L1) can be expressed as

$$
-q(G)=-q\left(G_{\mathcal{C}}^{\prime}\right)-q\left(G\left(B_{1}\right)\right)+1
$$

On the other hand, if $\operatorname{nlink}(G)$ represents the number of links on graph $G$, we have $n \operatorname{link}(G)=\operatorname{nlink}\left(G_{\mathcal{C}}^{\prime}\right)+\operatorname{nlink}\left(G\left(B_{1}\right)\right)$ and, from equation $(7), \operatorname{nlink}(G)-q(G)+1=$ $\operatorname{nlink}\left(G_{\mathcal{C}}^{\prime}\right)-q\left(G_{\mathcal{C}}^{\prime}\right)+1+\operatorname{nlink}\left(G\left(B_{1}\right)\right)-q\left(G\left(B_{1}\right)\right)+1$, i.e.,

$\operatorname{dim}(\operatorname{MGRP}(G))=\operatorname{dim}\left(\operatorname{MGRP}\left(G_{\mathcal{C}}^{\prime}\right)+\operatorname{dim}\left(\operatorname{MGRP}\left(G\left(B_{1}\right)\right)=K+Q+M\right.\right.$.

Note: Condition (L1) is always satisfied when $G_{\mathcal{C}}$ is a directed graph: after replacing a node $B_{i}$ by a graph $G\left(B_{i}\right)$, each edge-connected component in $G\left(B_{i}\right)$ is also an edgeconnected component in the resulting graph $G$, and $q(G)=q\left(G_{\mathcal{C}}\right)+q\left(G\left(B_{i}\right)\right)-1$ holds. For example, graphs $G_{\mathcal{C}}$ and $G$ shown in figure 3 (the edge-conected components of each $G\left(B_{i}\right)$ are represented by black circles), satisfy $q\left(G_{\mathcal{C}}\right)=3$ and $q(G)=6=$ $3+(2-1)+(3-1)$.

Nevertheless, when $G_{\mathcal{C}}$ is a mixed graph, after replacing a node $B_{i}$ by a graph $G\left(B_{i}\right)$, different edge-connected components in $G\left(B_{i}\right)$ can be connected by edges in the resulting graph $G$ and $q(G)<q\left(G_{\mathcal{C}}\right)+q\left(G\left(B_{i}\right)\right)-1$ would hold. In such a case, condition (L1) would not be satisfied. For example, graphs $G_{\mathcal{C}}$ and $G$ in figure 4 satisfy $q\left(G_{\mathcal{C}}\right)=1$ and $q(G)=3 \neq 1+(2-1)+(3-1)$, while those in figure 5 satisfy condition (L1) since $q\left(G_{\mathcal{C}}\right)=1$ and $q(G)=4=1+(2-1)+(3-1)$. However, note that if $G_{\mathcal{C}}$ has 
edges only between two given nodes, say $B_{i}$ and $B_{j}$, condition (L1) is satisfied if all the edges link the same edge-connected component of $G\left(B_{i}\right)$ to the same edge-connected component of $G\left(B_{j}\right)$.
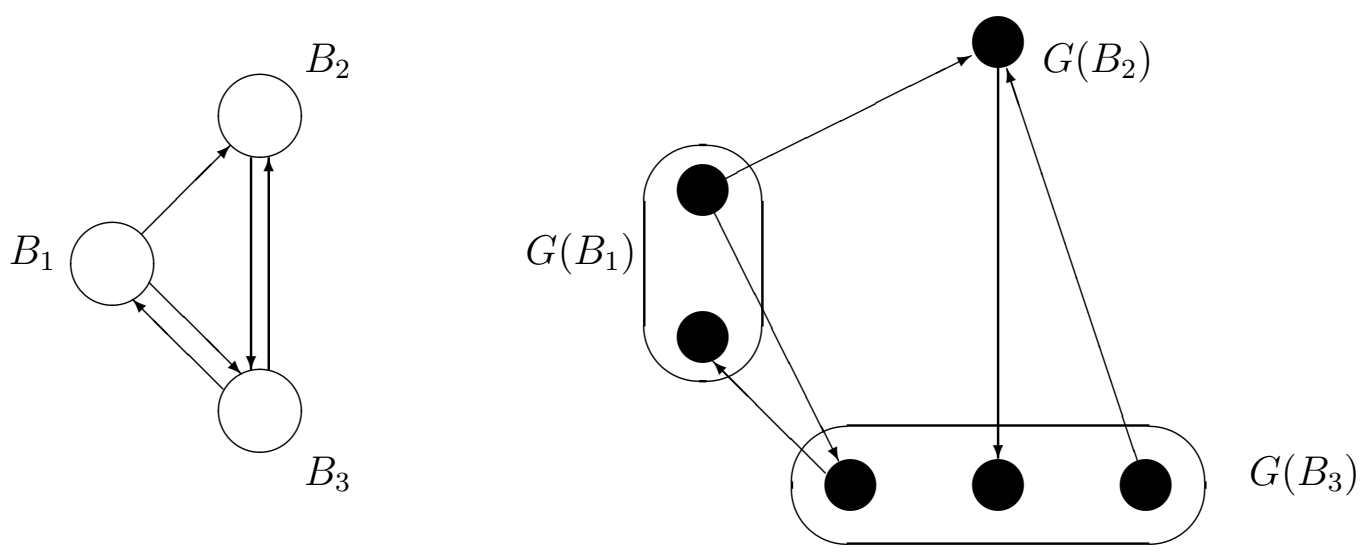

Figure 3: (L1) is satisfied when $G_{\mathcal{C}}$ is a directed graph.
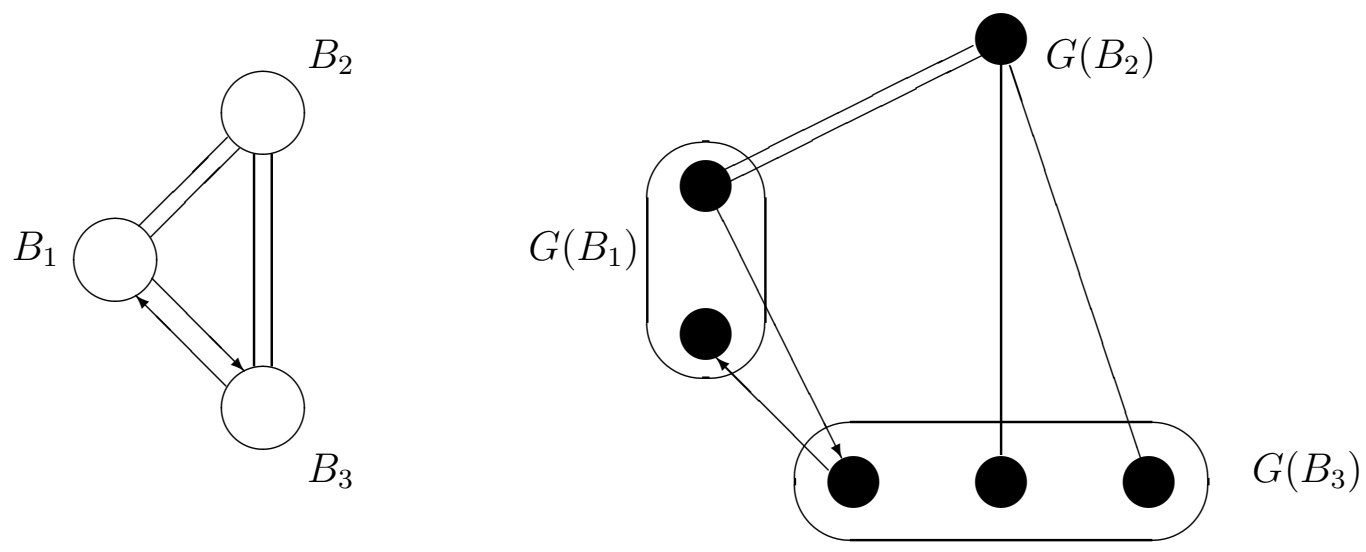

Figure 4: $G_{\mathcal{C}}$ is a mixed graph and (L1) is not satisfied.

When condition (L1) is not satisfied, the following second lifting theorem will be tried.

Theorem 5 Let $G$ be a mixed graph and let $\mathcal{C}$ be a configuration on $G$. The associated configuration inequality is facet-inducing for $M G R P(G)$ if the configuration inequality $F(x) \geq c_{0}$ associated with $\mathcal{C}$ on graph $G_{\mathcal{C}}$ is facet-inducing for $M G R P\left(G_{\mathcal{C}}\right)$ and the following conditions are satisfied:

L2(a) Given any proper subset of edges in $G_{\mathcal{C}}$, it is possible to replace each edge by an arc in such a way that inequality $F(x) \geq c_{0}$ is also facet-inducing for the MGRP polyhedron associated with the resulting graph, $G_{\mathcal{C}}^{\prime}$.

L2(b) Given any of the previous graphs $G_{\mathcal{C}}^{\prime}$ and given any arc a replacing an edge in $G_{\mathcal{C}}$, if we change arc a to an arc with the opposite direction, there exists at least one semitour $x^{*}$ for the MGRP on the resulting graph satisfying $F\left(x^{*}\right)=c_{0}$ 

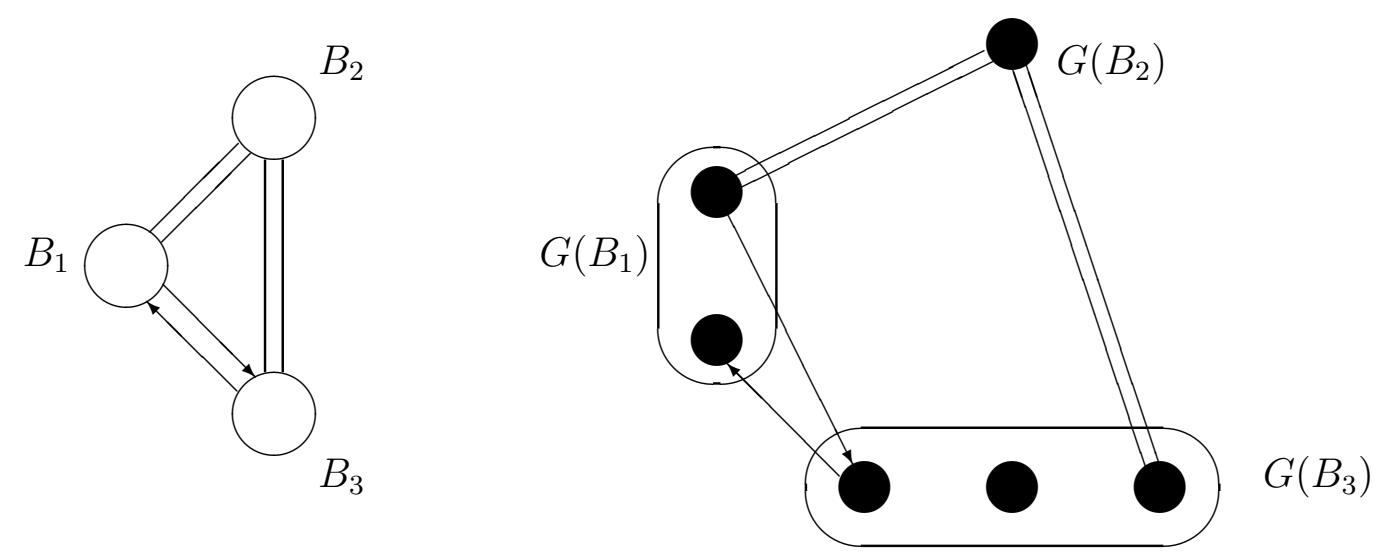

Figure 5: $G_{\mathcal{C}}$ is a mixed graph and (L1) is satisfied.

L2(c) Graph $G_{\mathcal{C}}$ has edges only between two given nodes, say $B_{1}$ and $B_{2}$.

Proof: Again, $F(x) \geq c_{0}$ will denote both the inequality on $G$ and on $G_{\mathcal{C}}$. Let us call $\omega=q\left(G_{\mathcal{C}}\right)+\sum_{i=1}^{r}\left(q\left(G\left(B_{i}\right)\right)-1\right)-q(G) \geq 0$, where $q(G)$ is defined as before. We will proceed by induction on the value of $\omega$. If $\omega=0$ then condition (L1) is satisfied and, from theorem $4, F(x) \geq c_{0}$ is facet inducing of $\operatorname{MGRP}(G)$. Assume that the result is true for every pair of graphs $G, G_{\mathcal{C}}$ with $\omega \leq n-1$ and let $G, G_{\mathcal{C}}$ be graphs with $\omega=n \geq 1$.

As, from L2(c), the only edges of graph $G_{\mathcal{C}}$ link $B_{1}$ and $B_{2}$, if $\omega \geq 1$ not all the edges in $G$ are linking the same edge-connected component of $G\left(B_{1}\right)$ to the same edgecomponent of $G\left(B_{2}\right)$, i.e., if we consider the bipartite graph in figure 6 having node sets formed by the edge-connected components of $G\left(B_{1}\right)$ and $G\left(B_{2}\right)$, say $\left\{S_{1}, S_{2}, \ldots, S_{m}\right\}$ and $\left\{T_{1}, T_{2}, \ldots, T_{n}\right\}$, and having an edge $\left(S_{i}, T_{j}\right)$ for each $E\left(S_{i}: T_{j}\right) \neq \emptyset$, then it has, at least, two edges. Consider a node that it is not a cut-vertex of this graph, say $S_{1}$ in $G\left(B_{1}\right)$. By construction, there exists another node, say $S_{2}$, also in $G\left(B_{1}\right)$, which is incident to some edges. If we delete from $G$ and from $G_{\mathcal{C}}$ the edges incident to $S_{1}$

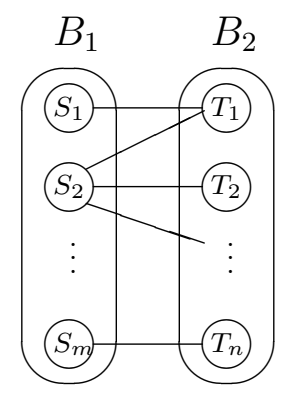

Figure 6: Bipartite graph used in the proof of theorem 5

(transforming them into arcs, for example), the resulting graphs $G^{\prime}$ and $G_{\mathcal{C}}^{\prime}$ satisfy, respectively, $q\left(G^{\prime}\right)=q(G)+1$ (because $S_{1}$ is not a cut-vertex) and $q\left(G_{\mathcal{C}}^{\prime}\right)=q\left(G_{\mathcal{C}}\right)$ (because $E\left(S_{2}: B_{2}\right) \neq \emptyset$ and $G_{\mathcal{C}}$ has edges only between $B_{1}$ and $\left.B_{2}\right)$. Hence, we have two graphs $G^{\prime}$ and $G_{\mathcal{C}}^{\prime}$ with $\omega=n-1$. From L2(a), we can assign a direction to 
each edge in $E\left(S_{1}\right)$ in such a way that inequality $F(x) \geq c_{0}$ is facet-inducing for the MGRP polyhedron associated with the resulting graph, $G_{\mathcal{C}}^{\prime}$. From the hypothesis of induction, $F(x) \geq c_{0}$ is also facet-inducing for the MGRP polyhedron associated with $G^{\prime}$. Let us assume that $c_{0} \neq 0$ (otherwise, the proof would be similar). Therefore, there exists nlink $\left(G^{\prime}\right)-q\left(G^{\prime}\right)+1$ linearly independent semitours for the MGRP on $G^{\prime}$ satisfying $F(x)=c_{0}$. Given that these semitours are also semitours for the MGRP on $G$ and given that $\operatorname{nlink}\left(G^{\prime}\right)=\operatorname{nlink}(G)$ and $q\left(G^{\prime}\right)=q(G)+1$, we have $n \operatorname{link}(G)$ $q(G)$ semitours and only one more tour is needed for $F(x) \geq c_{0}$ to be facet-inducing for $\operatorname{MGRP}(G)$.

Let $e$ be one of the edges of $G_{\mathcal{C}}$ in $E\left(S_{1}\right)$ that had been changed into an arc in $G_{\mathcal{C}}^{\prime}$. Let $G{ }^{\prime \prime} \mathcal{C}$ be the graph obtained by changing the arc $e$ into an arc with the opposite direction. From L2(b), there exists a semitour $x^{*}$ for the MGRP on $G^{\prime \prime} c$ satisfying $F\left(x^{*}\right)=c_{0}$. Given that $G\left(B_{i}\right)$ are strongly connected graphs, we can complete $x^{*}$ with links of $\cup \gamma\left(B_{i}\right)$ to obtain a semitour for the MGRP on $G$, also called $x^{*}$, satisfying $F\left(x^{*}\right)=c_{0}$. Finally, in order to show that $x^{*}$ is not a linear combination of the previous nlink $(G)-q(G)$ semitours, we only have to notice that these last semitours satisfy the equation $x\left(A^{+}\left(S_{1}\right)\right)+\left|A^{+}\left(S_{1}\right)\right|=x\left(A^{-}\left(S_{1}\right)\right)+\left|A^{-}\left(S_{1}\right)\right|$ considered in graph $G^{\prime}$, whilst $x^{*}$ satisfies equation $x\left(A^{+}\left(S_{1}\right) \backslash\{e\}\right)+\left|A^{+}\left(S_{1}\right)\right|-1=x\left(A^{-}\left(S_{1}\right)\right)+x_{e}+\left|A^{-}\left(S_{1}\right)\right|+1$ also considered in graph $G^{\prime}$.

\section{Basic inequalities}

Let us begin studying inequalities (4) and (5), which are valid for $\operatorname{MGRP}(G)$ and will be referred to as connectivity and balanced-set inequalities, respectively.

\subsection{Connectivity inequalities}

Theorem 6 : Inequalities (4), $x\left(A^{+}(S)\right) \geq 1, \forall S=\cup_{k \in Q} V_{k}, Q \subset\{1, \ldots, p\}$, are facet-inducing for $M G R P(G)$ if graphs $G(S)$ and $G(V \backslash S)$ are strongly connected.

Proof: The configuration graph $G_{\mathcal{C}}$ has only two nodes, say 1 and 2, corresponding to $S$ and to $V \backslash S$, respectively. As $E(S)=A_{R}(S)=\emptyset$, vertices 1 and 2 are joined by a pair of opposite non required arcs. Therefore, $\operatorname{dim}\left(\operatorname{MGRP}\left(G_{\mathcal{C}}\right)\right)=2-2+1=1$ and, since the semitour $x_{1,2}=x_{2,1}=1$ satisfies $x^{+}(S)=x_{1,2}=1$, the inequality is facet-inducing for $\operatorname{MGRP}\left(G_{\mathcal{C}}\right)$. Given that $(S: V \backslash S)$ does not contain edges, condition $(L 1)$ of the first lifting theorem 4 is satisfied and the inequality is also facet-inducing for $\operatorname{MGRP}(G)$.

Even if $G(S)$ is not strongly connected, there may exist a subset $S_{0} \subset S$ such that $G\left(S_{0}\right)$ is strongly connected, $S_{0}=\cup_{k \in Q^{\prime}} V_{k}, Q^{\prime} \subset Q$ and $A^{+}(S)=A^{+}\left(S_{0}\right)$. In this case, inequality (4) is facet inducing. Otherwise, it can be shown that inequality (4) is not facet inducing because it is dominated by a balanced-set inequality or by another connectivity inequality. 


\subsection{Balanced-set inequalities}

Theorem 7 : Let $S \subset V$ be such that $G(S)$ and $G(V \backslash S)$ are strongly connected. The balanced-set inequality (5), $-x\left(A^{+}(S)\right)+x\left(A^{-}(S)\right)+x(E(S)) \geq u_{S}$, where $u_{S}=$ $\left|A_{R}^{+}(S)\right|-\left|A_{R}^{-}(S)\right|-|E(S)|$, is facet-inducing for $M G R P(G)$ if and only if the following conditions are satisfied:

(i) $A^{+}(S) \neq \emptyset$

(ii) $E(S) \neq \emptyset$

(iii) $q(G)=q(G(S))+q(G(V \backslash S))-1$

Proof: The conditions are sufficient: Consider the corresponding graph $G_{\mathcal{C}}$ having only two vertices, say 1 and 2 , a number $\left|A_{R}^{+}(S)\right|$ of required (parallel) arcs from 1 to 2 , a number $\left|A_{R}^{-}(S)\right|$ of required arcs from 2 to 1 , a number $|E(S)|$ of edges and, possibly, a pair of opposite non required arcs. Condition (ii) implies that $q\left(G_{\mathcal{C}}\right)=1$ and then (iii) implies condition (L1) of the first lifting theorem. Hence, it suffices to show the result on graph $G_{\mathcal{C}}$, by finding an appropriate number of affinely independent semitours satisfying (5) to equality. This is equivalent to find $|E \cup A|$ affinely independent tours satisfying

$$
x\left(A^{+}(1)\right)=x\left(A^{-}(1)\right)+x(E(1))
$$

Notice that, in such tours, edges have to be traversed from vertex 2 to 1 . Hence, in what follows, we will not differentiate between edges (in $E(1))$ and $\operatorname{arcs}$ in $A^{-}(1)$. Let us call $K=\left|A^{+}(1)\right|$ and $M=\left|E(1) \cup A^{-}(1)\right|$. We study two cases:

(a) $K=M$. Consider the tour $x^{*}$ defined as $x_{e}^{*}=1$ for all $e \in A^{+}(1) \cup A^{-}(1) \cup E(1)$. Let now $e_{1}$ be a given link in $A^{-}(1) \cup E(1)$. For each arc $e \in A^{+}(1)$, let $x^{e}$ be defined as $x_{e}^{e}=x_{e_{1}}^{e}=2$ and $x_{a}^{e}=1$ for all $a \neq e, e_{1}$. Let now $a_{1}$ be a given arc in $A^{+}(1)$. For each link $e \in E(1) \cup A^{-}(1)$, let $x^{e}$ be defined as $x_{e}^{e}=x_{a_{1}}^{e}=2$ and $x_{a}^{e}=1$ for all $a \neq e, a_{1}$. We have defined $|E \cup A|+1$ different tours satisfying $x\left(A^{+}(1)\right)=x\left(A^{-}(1)\right)+x(E(1))$. Again, by arranging them as the rows of a matrix (figure 7(a)) and subtracting the row 1 (associated with $x^{*}$ ) from all the remaining rows, we obtain a matrix with rank $|E \cup A|-1$. Thus, $|E \cup A|$ of these tours are affinely independent.

(b) $K \neq M$. Let us assume, for example, that $K<M$ and denote $\sigma=M-K+1 \neq 1$. For each arc $e \in A^{+}(1)$, let $x^{e}$ be defined as $x_{e}^{e}=\sigma$ and $x_{a}^{e}=1$ for all $a \neq e$. Let $a_{1}$ be a given arc in $A^{+}(1)$. For each link $e \in E(1) \cup A^{-}(1)$, let $x^{e}$ be defined as $x_{e}^{e}=2, x_{a_{1}}^{e}=\sigma+1$ and $x_{a}^{e}=1$ for all $a \neq e, a_{1}$. These $|E \cup A|$ tours satisfy $x\left(A^{+}(1)\right)=x\left(A^{-}(1)\right)+x(E(1))$. Again, if we arrange them as the rows of a matrix (figure $7(\mathrm{~b})$ ) and we subtract the first row from all the remaining rows, we obtain a matrix with rank $|E \cup A|-1$.

The conditions are necessary:

(i) If $A^{+}(S)=\emptyset$, inequality (5) can be written as $x(E(S))+x\left(A^{-}(S)\right) \geq$ $-\left|A_{R}^{-}(S)\right|-|E(S)|$, that it is obviously dominated by the sum of several trivial inequalities.

(ii) If $E(S)=\emptyset$, inequality (5) becomes $x\left(A^{+}(S)\right)+u_{S} \leq x\left(A^{-}(S)\right)$ which is satisfied to equality (symmetry condition) by all the semitours for the MGRP on $G$. 


\begin{tabular}{c|cccc|cccc} 
& \multicolumn{5}{|c|}{$A^{+}(1)$} & \multicolumn{3}{|c}{$A^{-}(1) \cup E(1)$} \\
\hline$x^{*}$ & 1 & 1 & $\ldots$ & 1 & 1 & 1 & $\ldots$ & 1 \\
\hline & 2 & 1 & $\ldots$ & 1 & 2 & 1 & $\ldots$ & 1 \\
& 1 & 2 & $\ldots$ & 1 & 2 & 1 & $\ldots$ & 1 \\
& & $\ddots$ & & & & $\ldots$ & 1 \\
& & 1 & $\ldots$ & 2 & 2 & 1 & $\ldots$ & 1 \\
\hline & 2 & 1 & $\ldots$ & 1 & 2 & 1 & $\ldots$ & 1 \\
2 & 1 & $\ldots$ & 1 & 1 & 2 & $\ldots$ & 1 \\
& & & $\ldots$ & & & & $\ddots$ & \\
& 2 & 1 & $\ldots$ & 1 & 1 & 1 & $\ldots$ & 2 \\
\hline
\end{tabular}

(a)

\begin{tabular}{|cccc|cccc|}
\multicolumn{4}{c}{$A^{+}(1)$} & \multicolumn{1}{c}{$A^{-}(1) \cup E(1)$} \\
\hline$\sigma$ & 1 & $\ldots$ & 1 & 1 & 1 & $\ldots$ & 1 \\
1 & $\sigma$ & $\ldots$ & 1 & 1 & 1 & $\ldots$ & 1 \\
& & $\ddots$ & & & & $\ldots$ & 1 \\
1 & 1 & $\ldots$ & $\sigma$ & 1 & 1 & $\ldots$ & 1 \\
\hline$\sigma+1$ & 1 & $\ldots$ & 1 & 2 & 1 & $\ldots$ & 1 \\
$\sigma+1$ & 1 & $\ldots$ & 1 & 1 & 2 & $\ldots$ & 1 \\
& & $\ldots$ & & & & $\ddots$ & \\
$\sigma+1$ & 1 & $\ldots$ & 1 & 1 & 1 & $\ldots$ & 2 \\
\hline \multicolumn{6}{c}{} & \multicolumn{3}{c}{$(\mathrm{b})$} & & &
\end{tabular}

Figure 7: Matrices appearing in the proof of theorem 7

(iii) If $q(G)<q(G(S))+q(G(V \backslash S))$ - 1 , there exists a cut-set $\left(S_{0}: S_{1}\right)$ in $G(S)$ (or in $G(V \backslash S)$ ) such that $E\left(S_{0}: S_{1}\right)=\emptyset$ and $E\left(S_{0}: V \backslash S\right) \neq \emptyset \neq E\left(S_{1}: V \backslash S\right)$. It can be shown that the balanced-set inequality corresponding to set $S$ is obtained as the sum of the balanced-set inequalities corresponding to sets $S_{0}$ and $S_{1}$.

Note: Let $S \subset V$ be such that there exists a cut-set $\left(S_{0}: S_{1}\right)$ in $G(S)$ with $E\left(S_{0}\right.$ : $\left.S_{1}\right)=\emptyset, E\left(S_{0}: V \backslash S\right) \neq \emptyset$ and $E\left(S_{1}: V \backslash S\right)=\emptyset$. As before, it can be shown that the balanced-set inequality corresponding to $S$ is the sum of the balanced-set inequality corresponding to $S_{0}$ and the equation $x\left(A^{+}\left(S_{1}\right)\right)+u_{S_{1}}=x\left(A^{-}\left(S_{1}\right)\right)$. Hence, balanced-set inequalities associated with $S$ and $S_{0}$ are equivalent and, in practice, we could only consider balanced-set inequalities corresponding to sets $S$ such that $S \subset K_{i}$ for any edge-connected component $K_{i}$ of $G$. Notice also that, unlike the connectivity inequalities, the balanced-set inequalities corresponding to $S$ and $V \backslash S$ are not equivalent.

\section{$4.3 \quad$ R-odd cut inequalities}

A cut-set $\delta(S)$ will be called $R$-odd if it contains an odd number of required links or, equivalently, if both $S$ and $V \backslash S$ contain an odd number of $R$-odd vertices. Any tour must cross any given link cut-set an even number of times. This means that if $\delta(S)$ is $R$-odd, then the tour must traverse all their required links and, at least, one more link. Hence, any semitour must use, at least, one link in each $R$-odd cut-set and the following inequalities, which will be called $R$-odd cut inequalities, are valid for $\operatorname{MGRP}(G)$ :

$$
x(\delta(S)) \geq 1, \forall \delta(S) \quad R \text {-odd cut-set }
$$

Theorem 8 : Let $\delta(S)$ be an $R$-odd cut-set of $G$ such that $G(S)$ and $G(V \backslash S)$ are strongly connected. The $R$-odd cut inequality $(8), x(\delta(S)) \geq 1$, is facet-inducing for $M G R P(G)$ if and only if $|\alpha-\beta|<\epsilon$, where $\alpha=\left|A_{R}^{+}(S)\right|, \beta=\left|A_{R}^{-}(S)\right|$ and $\epsilon=|E(S)|$

Proof: The condition is necessary. If $|\alpha-\beta|=\epsilon$, then $\alpha+\beta+\epsilon=\left|\delta_{R}(S)\right|$ is not odd. If $|\alpha-\beta|>\epsilon$, the $R$-odd cut inequality is dominated by a balanced-set inequality. To show 
this, let us assume that $\alpha \geq \beta$. Then, $\alpha-\beta>\epsilon$ and therefore $\alpha-\beta-\epsilon=u_{S} \geq 1$. From the balanced-set inequality associated with $S, x\left(A^{-}(S)\right)+x(E(S)) \geq x\left(A^{+}(S)\right)+u_{S} \geq$ 1 holds and dominates (8).

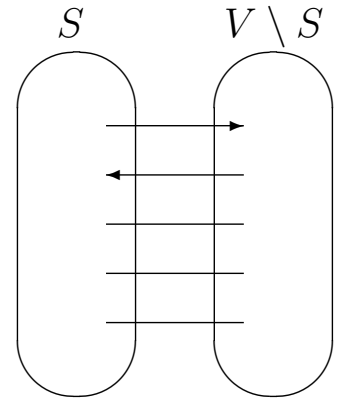

(a) Graph $G_{\mathcal{C}}$

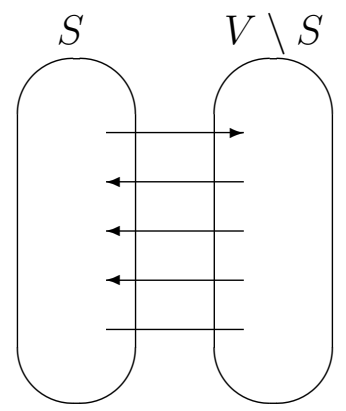

(c) Graph $G^{\prime \prime} \mathrm{c}$

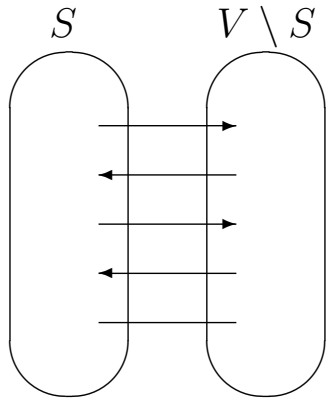

(b) Graph $G_{\mathcal{C}}^{\prime}$

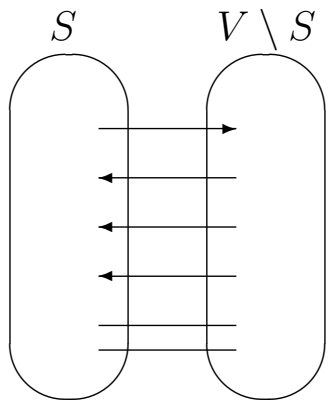

(d) Tour $x^{*}+x^{R}$

Figure 8: Graphs $G_{\mathcal{C}}, G_{\mathcal{C}}^{\prime}$ and $G^{\prime \prime}{ }_{\mathcal{C}}$ and tour $x^{*}+x^{R}$ used in the proof of theorem 8.

The condition is sufficient. We first show the result for the graph $G_{\mathcal{C}}$. This graph has two vertices, say 1 and 2, a number $\alpha$ of required (parallel) arcs from 1 to 2 , a number $\beta$ of required arcs from 2 to 1 , a positive number $\epsilon$ of edges and, possibly, a pair of opposite non required arcs. Hence, $\operatorname{dim}\left(\operatorname{MGRP}\left(G_{\mathcal{C}}\right)\right)=n \operatorname{link}\left(G_{\mathcal{C}}\right)$. Condition $\epsilon \geq|\alpha-\beta|+1$ implies that there are enough edges in $G_{\mathcal{C}}$ to balance a copy of every required arc and even one more. Hence, the following vectors are semitours for the MGRP on $G_{\mathcal{C}}$ :

- For each link $e$ (required or not), let $x^{e}$ be defined as $x_{e}^{e}=1$ and $x_{a}^{e}=0$ for each link $a \neq e$.

We have defined a number $n \operatorname{link}\left(G_{\mathcal{C}}\right)$ of linearly independent semitours satisfying $x(\delta(S))=1$. Hence, the $R$-odd cut inequality is facet-inducing for $\operatorname{MGRP}\left(G_{\mathcal{C}}\right)$, provided that $\epsilon \geq|\alpha-\beta|+1$. In order to show that it is also facet-inducing for $\operatorname{MGRP}(G)$, we will prove that condition (L2) of the second lifting theorem 5 is satisfied:

- L2(a) Given that $\epsilon \geq|\alpha-\beta|+1$ holds in $G_{\mathcal{C}}$, for any proper subset of edges in $G_{\mathcal{C}}$ it will always be possible to assign a direction to each edge in such a way that $\epsilon^{\prime} \geq\left|\alpha^{\prime}-\beta^{\prime}\right|+1$ is also satisfied in the resulting graph $G_{\mathcal{C}}^{\prime}$ (see figure 8). Hence, as it has been proved before, the $R$-odd cut inequality is facet-inducing for $\operatorname{MGRP}\left(G_{\mathcal{C}}^{\prime}\right)$. 
- L2(b) Let us now assume that in one of the previous graphs $G_{\mathcal{C}}^{\prime}$ with $\epsilon^{\prime}=\left|\alpha^{\prime}-\beta^{\prime}\right|+1$ we change the direction of one arc obtaining a graph $G^{\prime \prime} \mathcal{C}$ where $\epsilon "=|\alpha "-\beta "|-1$ in the worst case. Consider the semitour $x^{*}$ on $G^{\prime \prime}{ }_{\mathcal{C}}$ defined as $x_{e}^{*}=1$, where $e$ is an arbitrary edge and $x_{a}^{*}=0$ for the remaining links $a \neq e$ (see figure 8). This semitour satisfies $x(\delta(S))=1$ and $x^{*}+x^{R}$ satisfies the balanced-set condition, since it has $\epsilon "+1$ edges and a difference in $\operatorname{arcs}$ of $|\alpha "-\beta "|$, with $\epsilon "+1 \geq|\alpha "-\beta "|$.

- L2(c) Obvious.

If $G(S)$ (or $G(V \backslash S)$ ) is not connected, the $R$-odd cut inequality $x(\delta(S)) \geq 1$ is not facet-inducing: let $G\left(S_{0}\right)$ be a connected component of $G(S)$ such that $\delta\left(S_{0}\right)$ is also $R$-odd; then, the $R$-odd cut inequality corresponding to $S$ is dominated by the $R$-odd cut inequality corresponding to $S_{0}$. If $G(S)$ is connected but it is not strongly connected, $R$-odd cut inequalities may be or not facet-inducing.

\section{Path-Bridge inequalities}

Path-Bridge inequalities were introduced by Letchford (1997) for the undirected GRP. In this section we will generalize these inequalities to the MGRP.

A Path-Bridge configuration (figure 9) will be defined by two integers $P$ and $B$ with $P \geq 1, B \geq 0, P+B \geq 3$ and odd, by $n_{i} \geq 2$ integers, $i=1,2, \ldots, P$, by a partition of $V$ into subsets $\left\{A, Z, M_{j}^{i}: i=1,2, \ldots P, j=1,2, \ldots n_{i}\right\}$ and by two cost functions. The partition must satisfy that each $R$-set $V_{i}$ is contained in exactly one of the node sets $A \cup Z, M_{j}^{i}, i=1,2, \ldots P, j=1,2, \ldots n_{i}$ (i.e., each required link either lies in some $\gamma\left(M_{j}^{i}\right)$ or crosses from $A$ to $\left.Z\right)$, the induced subgraphs $G\left(M_{j}^{i}\right)$, $i=0,1, \ldots, P, j=1,2, \ldots n_{i}+1$, are strongly connected (where, for convenience, for all $i$ we identify $M_{0}^{i}$ with $A$ and $M_{n_{i}+1}^{i}$ with $\left.Z\right)$ and $(A: Z)$ contains a number $B$ of required links. Cost functions are defined as:

$$
\begin{aligned}
& c^{A}(A, Z)=c^{A}(Z, A)=c^{E}(A, Z)=1 \\
& c^{A}\left(M_{j}^{i}, M_{q}^{i}\right)=\frac{|j-q|}{n_{i}-1}, \quad \forall j, q \in\left\{0,1,2, \ldots, n_{i}+1\right\}, \quad 0<|j-q|<n_{i}+1 \\
& c^{A}\left(M_{j}^{i}, M_{q}^{r}\right)=\frac{1}{n_{i}-1}+\frac{1}{n_{r}-1}+\left|\frac{j-1}{n_{i}-1}-\frac{q-1}{n_{r}-1}\right|, \\
& \forall i, r \in\{1,2, \ldots, P\}, i \neq r, \quad j \in\left\{1,2, \ldots, n_{i}\right\}, q \in\left\{1,2, \ldots, n_{r}\right\}
\end{aligned}
$$

Furthermore, we will assume that:

- Sets $A\left(M_{j}^{i}: M_{j+1}^{i}\right)$ and $A\left(M_{j+1}^{i}: M_{j}^{i}\right), i=1,2, \ldots, P, \quad j=0,1, \ldots, n_{i}$, are nonempty. We will denote the set of such arcs by $\mathcal{E} x$ (external arcs).

- Sets $A\left(M_{j}^{i}: M_{q}^{i}\right), i=1,2, \ldots, P,|j-q|>1,\{j, q\} \neq\left\{0, n_{i}+1\right\}$, could be empty or not. We will denote the set of such arcs by $\mathcal{I} n$ (internal arcs). - Sets $A\left(M_{j}^{i}: M_{q}^{r}\right), i, r \in\{1,2, \ldots, P\}, i \neq r, \quad j \in\left\{1,2, \ldots, n_{i}\right\}, q \in$ $\left\{1,2, \ldots, n_{r}\right\}$, could be empty or not. We will denote the set of such arcs by $\mathcal{C} r$ (crossing arcs). 
The partition $\mathcal{B}=\left\{A, Z, M_{j}^{i}: i=1,2, \ldots P, j=1,2, \ldots n_{i}\right\}$ and the functions $c^{A}, c^{E}$ define the configuration graph $G_{\mathcal{C}}$ whose skeleton is showed in figure 9 . It has $P$ paths from $A$ to $Z$, each of them with $n_{i}+2$ nodes and $n_{i}+1$ pairs of opposite external arcs. Internal arcs $\left(M_{j}^{i}, M_{q}^{i}\right)$, not represented in figure 9 , have a cost equal to the length of the shortest path from $M_{j}^{i}$ to $M_{q}^{i}$ using external arcs.

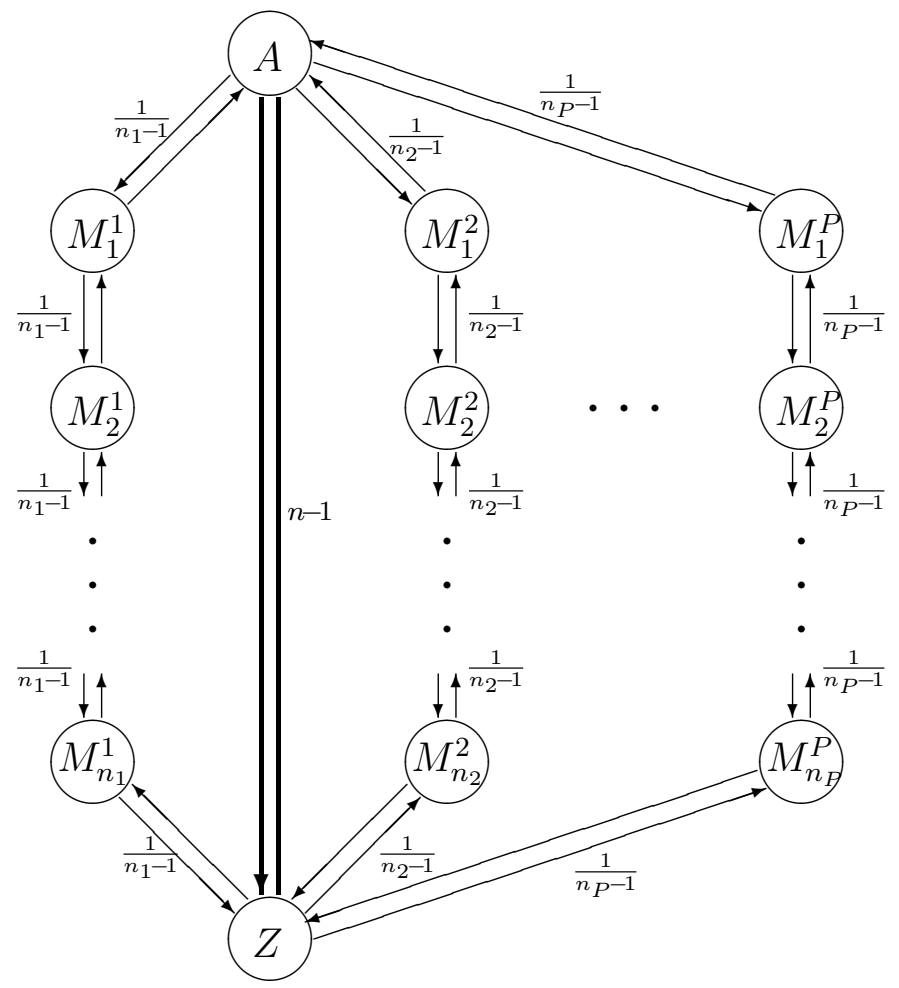

Figure 9: Path-Bridge configuration.

The Path-Bridge inequality corresponding to this configuration is defined by:

$$
\sum_{e \in E \cup A} c_{e} x_{e} \geq 1+\sum_{i=1}^{P} \frac{n_{i}+1}{n_{i}-1}
$$

We can define the $n$-regular Path-Bridge inequality in which $n_{i}$ for each path is the same number $n$. In this case, by multiplying by $n-1$, the inequality (9) can be rewritten in the following way:

$$
\sum_{e \in E \cup A} c_{e} x_{e} \geq P n+P+n-1
$$

where

$$
\begin{array}{ll}
c^{A}(A, Z)=c^{A}(Z, A)=c^{E}(A, Z)=n-1 & \\
c^{A}\left(M_{j}^{i}, M_{q}^{i}\right)=|j-q|, & \forall j, q \in\{0,1,2, \ldots, n+1\}, \quad 0<|j-q|<n+1 \\
c^{A}\left(M_{j}^{i}, M_{q}^{r}\right)=|j-q|+2 & \forall i, r \in\{1,2, \ldots, P\}, i \neq r, \quad j, q \in\{1,2, \ldots, n\}
\end{array}
$$


Again, internal $\operatorname{arcs}\left(M_{j}^{i}, M_{q}^{i}\right)$ have a cost equal to the length of the shortest path from $M_{j}^{i}$ to $M_{q}^{i}$ using external arcs. Crossing $\operatorname{arcs}\left(M_{j}^{i}, M_{q}^{r}\right), i \neq r \quad j, q \in\{1,2, \ldots, n\}$ have now a cost equal to their 'difference in level', $|i-j|$, plus two.

These inequalities will be also called Path-Bridge (PB) because they can be considered as the mixed version of the Path-Bridge inequalities for the undirected GRP (Letchford, 1997). These last inequalities include the Path inequalities for the (undirected) Graphical TSP, GTSP, proposed by Cornuèjols, Fonlupt \& Naddef (1985). It is worthwhile to point out that all these inequalities have the same coefficients and RHS.

$P B$ inequalities reduce to the $s$-Path inequalities for the GATSP, introduced by Chopra \& Rinaldi (1996), when there are no required links crossing from $A$ to $Z$ ( $B=0$ and, hence, $P \geq 3$ and odd). In such a case, sets $A$ and $Z$ are allowed to be empty and the inequalities are called $s$-Wheelbarrow and s-Bicycle inequalities. However, in general, the set $(A: Z)$ can contain one or more required links $(B>0)$.

Given that Path-Bridge inequalities are proved to be valid for the undirected GRP, they are also valid for $\operatorname{MGRP}(G)$. In order to show that, under certain conditions, they are facet-inducing of $\operatorname{MGRP}(G)$, we have to consider several cases: $P=1, P \geq 3$ and odd, $P \geq 2$ and even.

When $P=1$, the PB configuration becomes the so called $K-C$ configuration (figure 10). It is defined by an integer $K \geq 3$, a partition of $V$ into $K+1$ subsets $\left\{M_{0}, M_{1}, M_{2}, \ldots, M_{K-1}, M_{K}\right\}$ such that each $R$-set $V_{i}, 1 \leq i \leq p$, is contained in exactly one of the node sets $M_{0} \cup M_{K}, M_{1}, M_{2}, \ldots, M_{K-1}$, the induced subgraphs $G\left(M_{i}\right)$, $i=0,1,2, \ldots, K$, are strongly connected and $\left(M_{0}: M_{K}\right)$ contains a positive and even number of required links, and by the cost functions defined as:

$$
\begin{aligned}
& c^{A}\left(M_{0}, M_{K}\right)=c^{A}\left(M_{K}, M_{0}\right)=c^{E}\left(M_{0}, M_{K}\right)=K-2 \\
& c^{A}\left(M_{i}, M_{j}\right)=|i-j|, \quad \forall i, j:\{i, j\} \neq\{0, K\}
\end{aligned}
$$

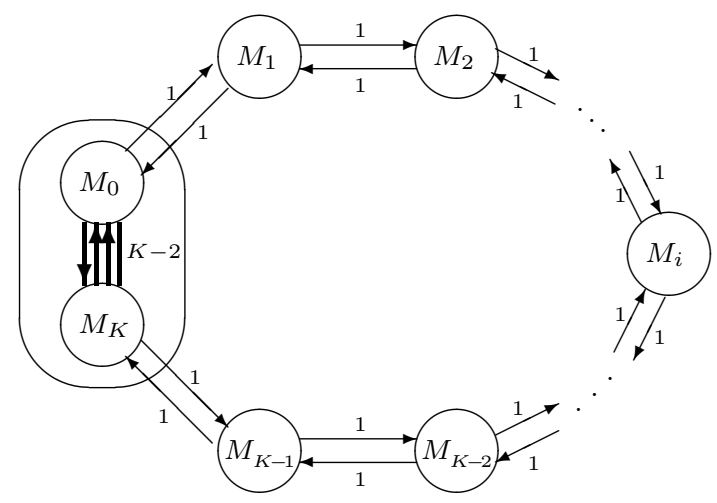

Figure 10: K-C configuration.

Note that $M_{0}$ and $M_{K}$ correspond to $A$ and $Z$ in the general $\mathrm{PB}$ configuration and $K-1$ is exactly $n_{1}$. 
The partition $\mathcal{B}=\left\{M_{0}, M_{1}, M_{2}, \ldots, M_{K-1}, M_{K}\right\}$ and the functions $c^{A}, c^{E}$ define the configuration graph $G_{\mathcal{C}}$ whose skeleton is showed in figure 10. Internal $\operatorname{arcs}\left(M_{i}, M_{j}\right)$ not represented in figure 10 have a cost equal to the length of the shortest path from $M_{i}$ to $M_{j}$ using external arcs. The associated $K$ - $C$ inequality is:

$$
(K-2) x\left(\left(M_{0}: M_{K}\right)\right)+\sum_{\substack{0 \leq i<j \leq K \\(i, j) \neq(0, K)}}|i-j| x\left(\left(M_{i}: M_{j}\right)\right) \geq 2(K-1)
$$

Constraints (11) are called K-C inequalities because they are identical to the K-C inequalities for the Undirected GRP (Corberán and Sanchis, 1994,1998). The effect of $\mathrm{K}-\mathrm{C}$ inequalities is to separate 'solution' vectors $x^{*}$ as shown in figure 11 , where $x_{e}^{*}=1$ for non required arcs (in thin lines) and $x_{e}^{*}=0$ for the required links (in bold lines). This vector satisfies all the facet-inducing inequalities described in previous sections but violates the $\mathrm{K}-\mathrm{C}$ inequality $F(x) \geq 6$ since $F\left(x^{*}\right)=4$.

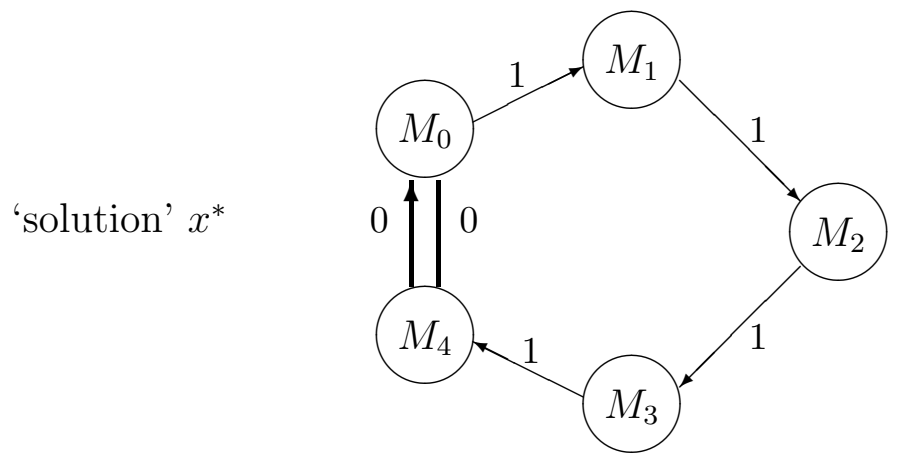

Figure 11: Vector violating a K-C inequality.

Theorem $9 \mathrm{~K}-C$ inequalities ( $P B$ inequalities with $P=1$ ) are facet-inducing for $\operatorname{MGRP}(G)$ if $\epsilon \geq|\alpha-\beta|$, where $\alpha=\left|A_{R}\left(M_{K}: M_{0}\right)\right|, \beta=\left|A_{R}\left(M_{0}: M_{K}\right)\right|$ and $\epsilon=\left|E\left(M_{0}: M_{K}\right)\right|$.

Proof: Let $F(x) \geq 2(K-1)$ denote the K-C inequality. We will consider two cases: $\epsilon=0$ and $\epsilon \geq 1$. Let us assume first that $\epsilon=0$ and, hence, $\alpha=\beta$. We will show first that $F(x) \geq 2(K-1)$ is facet-inducing for $\operatorname{MGRP}\left(G_{\mathcal{C}}\right)$. Given that $\epsilon=0$, graph $G_{\mathcal{C}}=\left(\mathcal{B}, A_{\mathcal{C}}\right)$ is a directed graph with $K+1$ edge-connected components. Then, $\operatorname{dim}\left(\operatorname{MGRP}\left(G_{\mathcal{C}}\right)\right)=\left|A_{\mathcal{C}}\right|-K$. The following vectors are semitours for the MGRP on $G_{\mathcal{C}}$ and satisfy $F(x)=2(K-1)$ :

(a) Semitours formed by all the pairs of external opposite arcs linking $M_{0}$ and $M_{1}, M_{1}$ and $M_{2}, \ldots, M_{K-1}$ and $M_{K}$ except one of them (figure 12(a)).

(b) For each arc $a \in A\left(M_{K}: M_{0}\right)$, required or not, consider the semitour $x$ consisting of arc $a$ and the arcs in the path $M_{0}, M_{1}, \ldots, M_{K}$ (figure 12(b)). In the same way, for each arc $a \in A\left(M_{0}: M_{K}\right)$ consider the semitour obtained by adding to $a$ the arcs in the path $M_{K}, M_{K-1}, \ldots, M_{1}, M_{0}$.

(c) For each arc $a=\left(M_{i}, M_{j}\right) \in \mathcal{I} n$ consider the semitour obtained by adding to $a$ the arcs in the path from $M_{j}$ to $M_{i}$ using external arcs and the pairs of $\operatorname{arcs}\left(M_{t}, M_{t+1}\right)$ and $\left(M_{t+1}, M_{t}\right), t \in\{0,1, \ldots, i-1, j, j+1, \ldots, K-1\}$ except one of them (figure $12(\mathrm{c})$ ). 

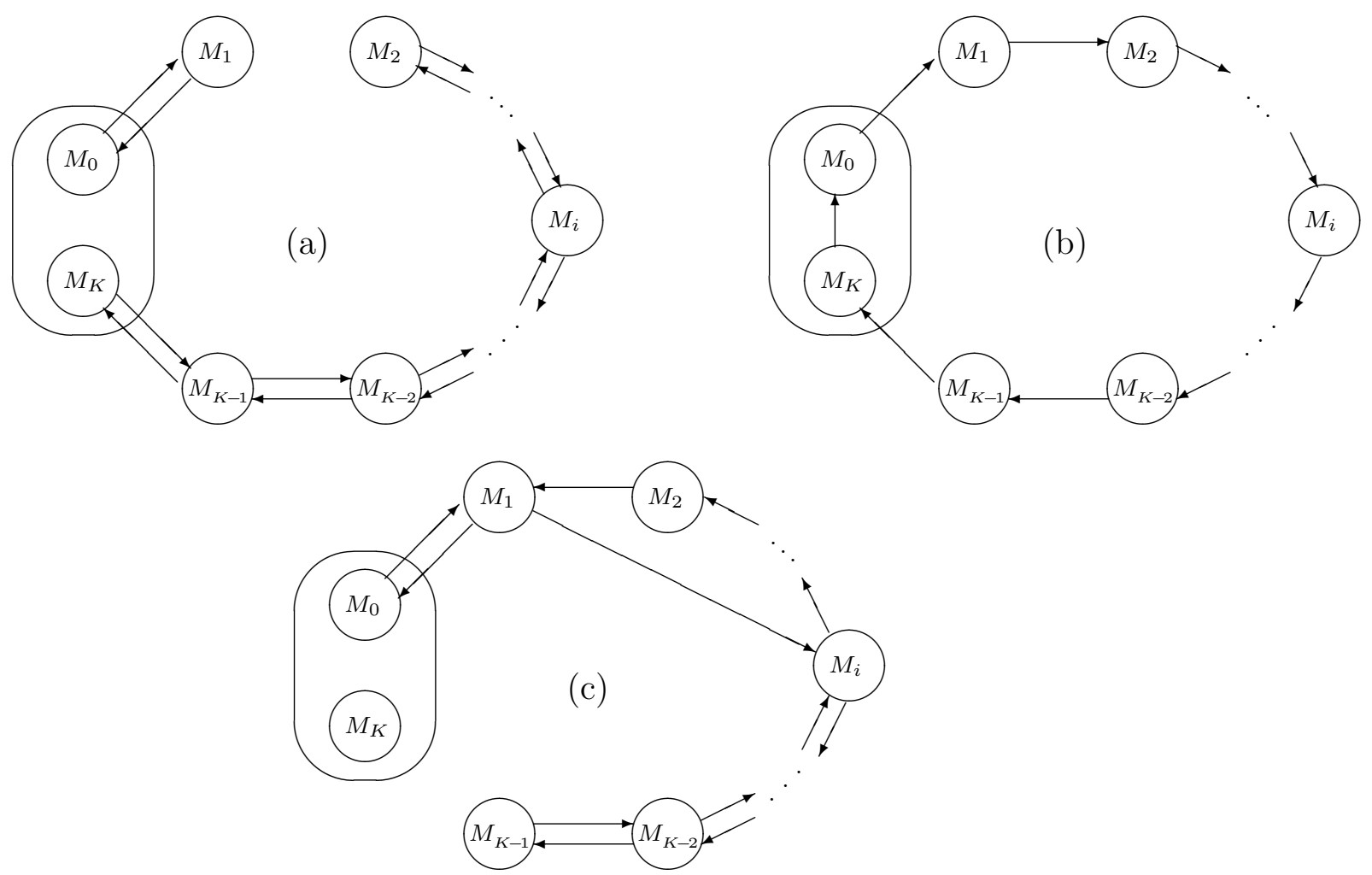

Figure 12: Semitours defined in the proof of theorem 9.

We have defined $K+\left|\left(M_{0}: M_{K}\right)\right|+|\mathcal{I} n|=|\mathcal{E} x|-K+\left|\left(M_{0}: M_{K}\right)\right|+|\mathcal{I} n|=\left|A_{\mathcal{C}}\right|-K$ semitours all satisfying $F(x)=2(K-1)$. If we express these semitours as rows of a matrix (sorted as (c),(b) and (a) before) and the arcs as columns (sorted as $\mathcal{I} n,\left(M_{0}: M_{K}\right)$ and $\left.\mathcal{E} x\right)$, we obtain the matrix showed in figure 13 . The block $B$, corresponding to rows (a) and columns $\left(M_{0}, M_{1}\right),\left(M_{1}, M_{2}\right), \ldots,\left(M_{K-1}, M_{K}\right)$, has full rank. Hence, the $\left|A_{\mathcal{C}}\right|-K$ semitours are affinely independent and $F(x) \geq 2(K-1)$ is facet-inducing for $\operatorname{MGRP}\left(G_{\mathcal{C}}\right)$. Furthermore, as the configuration graph $G_{\mathcal{C}}$ has no edges, condition (L1) of lifting theorem 4 is satisfied and, therefore, $F(x) \geq 2(K-1)$ is also facet-inducing for $\operatorname{MGRP}(G)$.

Let us assume now that $\epsilon \geq 1$ and, hence, $\operatorname{dim}\left(\operatorname{MGRP}\left(G_{\mathcal{C}}\right)=\left|E_{\mathcal{C}} \cup A_{\mathcal{C}}\right|-K+1\right.$. Similarly to the case $\epsilon=0$, we can define such a number of affinely independent semitours for the MGRP on $G_{\mathcal{C}}$ satisfying $F(x)=2(K-1)$. Therefore, the inequality is facet-inducing for $\operatorname{MGRP}\left(G_{\mathcal{C}}\right)$. In order to show that it is also facet-inducing for $\operatorname{MGRP}(G)$, it can be proved, as in theorem 8, that condition (L2) of lifting theorem 5 is satisfied.

Theorem $10 P B$ inequalities with $P \geq 3$ and odd are facet-inducing for $M G R P(G)$ if $\epsilon \geq|\alpha-\beta|$, where $\alpha=\left|A_{R}(A: Z)\right|, \beta=\left|A_{R}(Z: A)\right|$ and $\epsilon=|E(A: Z)|$.

Proof: Let $F(x) \geq c_{0}$ denote the PB inequality. We will consider two cases: $\epsilon=0$ and $\epsilon \geq 1$. Let us assume first that $\epsilon=0$ and, hence, $\alpha=\beta$. We will show first that 


\begin{tabular}{|c|c|c|}
\hline $\mathcal{I} n$ & $\left(M_{o}: M_{K}\right)$ & $\mathcal{E} x$ \\
\hline $\mathrm{I}$ & 0 & $*$ \\
\hline 0 & I & $*$ \\
\hline 0 & 0 & B B \\
\hline
\end{tabular}

$$
B=\left(\begin{array}{lllll}
0 & 1 & & 1 & 1 \\
1 & 0 & & 1 & 1 \\
& & \ddots & & \\
1 & 1 & & 0 & 1 \\
1 & 1 & & 1 & 0
\end{array}\right)
$$

Figure 13: Matrices appearing in the proof of theorem 9

$F(x) \geq c_{0}$ is facet-inducing for $\operatorname{MGRP}\left(G_{\mathcal{C}}\right)$. Given that $\epsilon=0$, graph $G_{\mathcal{C}}=\left(\mathcal{B}, A_{\mathcal{C}}\right)$ is a directed graph. Consider the GATSP instance obtained from graph $G_{\mathcal{C}}$ by making all arcs non-required. Then, the corresponding s-Path inequality defines a facet of $\operatorname{GATSP}\left(G_{\mathcal{C}}\right)$ (Chopra \& Rinaldi, 1996). As $\alpha=\beta$, every GATSP tour satisfying the s-Path inequality as an equality is also a semitour for the MGRP on $G_{\mathcal{C}}$. Given that $\operatorname{dim}\left(\operatorname{MGRP}\left(G_{\mathcal{C}}\right)\right)=\left|A_{\mathcal{C}}\right|-|\mathcal{B}|+1=\operatorname{dim}\left(\operatorname{GATSP}\left(G_{\mathcal{C}}\right)\right)$, the s-Path inequality is facet-inducing for $\operatorname{MGRP}\left(G_{\mathcal{C}}\right)$. Furthermore, as the configuration graph $G_{\mathcal{C}}$ has no edges, condition (L1) of lifting theorem 4 is satisfied and, therefore, $F(x) \geq c_{0}$ is also facet-inducing for $\operatorname{MGRP}(G)$ in this case.

Let us assume now that $\epsilon \geq 1$. Given that $B$ is even and $\epsilon \geq|\alpha-\beta|$, the $\epsilon$ edges in $G_{\mathcal{C}}$ can be oriented as arcs to obtain a new directed graph $G_{\mathcal{C}}^{\prime}$ satisfying $\alpha^{\prime}=\beta^{\prime}$ (and $\left.\epsilon^{\prime}=0\right)$. Then, we can find $\operatorname{dim}\left(\operatorname{MGRP}\left(G_{\mathcal{C}}^{\prime}\right)\right)$ semitours for the MGRP on $G_{\mathcal{C}}^{\prime}$ (and on $G_{\mathcal{C}}$ ) satisfying $F(x)=c_{0}$. Given that $\epsilon \geq 1$,

$$
\operatorname{dim}\left(\operatorname{MGRP}\left(G_{\mathcal{C}}\right)\right)=\left|A_{\mathcal{C}}\right|-(|\mathcal{B}|-1)+1=\operatorname{dim}\left(\operatorname{MGRP}\left(G_{\mathcal{C}}^{\prime}\right)\right)+1,
$$

and we need an extra semitour for the MGRP on $G_{\mathcal{C}}$. Let $e \in(A: Z)$ and consider the GATSP instance $G$ " ${ }_{\mathcal{C}}$ obtained from graph $G_{\mathcal{C}}$ by deleting all the links in $(A: Z)$ except the edge $e$, which is transformed into an arc $a$ with the opposite direction to that associated to $e$ in $G_{\mathcal{C}}^{\prime}$. It is known that there exists a GATSP tour $x^{*}$ on $G$ " $\mathcal{C}$ using only external arcs and exactly one copy of $a$ and satisfying the s-Path inequality as an equality. By adding to $x^{*}$ the original required links in $(A: Z)$, as $\epsilon \geq|\alpha-\beta|$, we obtain a (balanced) tour for the MGRP on $G_{\mathcal{C}}$. Then, $x^{*}$ is a semitour for the MGRP on $G_{\mathcal{C}}$. Furthermore, $x^{*}$ is linearly independent with the previously mentioned semitours due to the different orientation of edge $e$. Therefore, the inequality $F(x) \geq c_{0}$ is facet-inducing for $\operatorname{MGRP}\left(G_{\mathcal{C}}\right)$. In order to show that it is also facet-inducing for $\operatorname{MGRP}(G)$, it is not difficult to see that, as in theorem 8, condition (L2) of lifting theorem 5 is satisfied.

The effect of $\mathrm{PB}$ inequalities for $P$ even is to separate 'solutions' as that shown in figure 14. It can be seen that vector $x^{*}$, where $x_{e}^{*}=1$ for the non required arcs (in thin lines) and $x_{e}^{*}=0$ for the required link (in bold line), satisfies all the facet- 
inducing inequalities described in previous sections, but violates the regular $\mathrm{PB}$ inequality $F(x) \geq 7$ since $F\left(x^{*}\right)=6$.

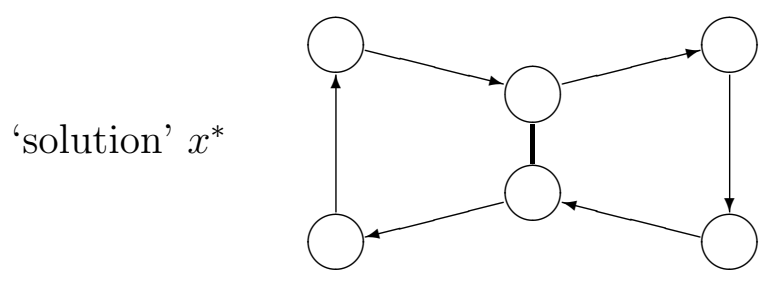

Figure 14: Vector violating a 2-PB inequality.

Theorem $11 P B$ inequalities with $P$ even are facet-inducing for $M G R P(G)$ if $\epsilon \geq$ $|\alpha-\beta|+1$, where $\alpha=\left|A_{R}(A: Z)\right|, \beta=\left|A_{R}(Z: A)\right|$ and $\epsilon=|E(A: Z)|$.

Proof: Let $F(x) \geq c_{0}$ denote the $\mathrm{PB}$ inequality. Again, we will show first that $F(x) \geq c_{0}$ is facet-inducing for $\operatorname{MGRP}\left(G_{\mathcal{C}}\right)$. Given that $\epsilon \geq 1$,

$$
\begin{aligned}
\operatorname{dim}\left(\operatorname{MGRP}\left(G_{\mathcal{C}}\right)=\right. & |(A: Z) \cup \mathcal{I} n \cup \mathcal{C} r|+2 \sum\left(n_{i}+1\right)-\left(\sum n_{i}+1\right)+1= \\
& =|(A: Z) \cup \mathcal{I} n \cup \mathcal{C} r|+\sum\left(n_{i}+2\right)
\end{aligned}
$$

and this is the number of linearly independent semitours satisfying $F(x)=c_{0}$ we have to find.

As for the Path inequalities for the GTSP (see Cornuèjols et al., 1985), it can be seen that for each link $e \in(A: Z) \cup \mathcal{I} n \cup \mathcal{C} r$, there always exists a semitour for the MGRP in $G_{\mathcal{C}}$ satisfying $F(x)=c_{0}$ and using $e$ exactly once and external arcs. Hence, only $\sum\left(n_{i}+2\right)$ more linearly independent semitours, using only external arcs, are needed.

(a) For each path $i^{*} \in\{1,2, \ldots, P\}$ and for each $j^{*} \in\left\{0,1,2, \ldots, n_{i^{*}}\right\}$, we consider the semitour that uses the two opposite arcs in each $\left(B_{j}^{i^{*}}: B_{j+1}^{i^{*}}\right)$, except for $j=j^{*}$, and one single arc in each $\left(B_{j}^{i}: B_{j+1}^{i}\right), i \neq i^{*}$, in such a way that $\frac{p}{2}$ paths are oriented from $A$ to $Z$ and the remaining $\frac{p}{2}-1$ paths are oriented from $Z$ to $A$. We will assume that these $n_{i^{*}}+1$ semitours use the same orientation for the other $P-1$ paths. In other words, $P-1$ of the paths joining $A$ and $Z$ are used once, while the last path is broken into two parts and each part is used twice. These sets of arcs are semitours for the MGRP on $G_{\mathcal{C}}$ because, given that $\epsilon \geq|\alpha-\beta|+1$, by adding to them the required links we obtain an eulerian graph. All these semitours satisfy $F(x)=c_{0}$.

(b) For each path $i$, it is possible to build $n_{i}+1$ different semitours using the opposite orientations to those used in (a) for the other $P-1$ paths. Among them we select just the semitour corresponding to the first of those described in (a) for each $i$.

Therefore, we have $n_{i}+2$ semitours for the MGRP, satisfying $F(x)=c_{0}$, associated to each path $i$. In order to show that these $\sum\left(n_{i}+2\right)$ semitours are linearly independent, consider their incidence matrix relative to the external arcs. In this matrix, the columns correspond to the external arcs and are grouped by paths. Columns associated to arcs 
in each path are sorted in the following way: the first half correspond to the arcs oriented from $A$ to $Z$ and the second one to those oriented from $Z$ to $A$.

By expressing the semitours as rows of this matrix (sorted as (a) and (b) before), we obtain the matrix showed in figure 15. Submatrix $B\left(\left(n_{i}+1\right) \times\left(n_{i}+1\right)\right)$ is shown in figure $13,[k]$ represents a $\left(n_{i}+1\right) \times\left(n_{j}+1\right)$ matrix with all its entries equal to $k,(k)$ represents a vector with $n_{i}+1$ components equal to $k$ and $B_{1}$ is the first row of matrix $B$.

\begin{tabular}{|c|c|c|c|c|c|c|c|c|c|c|c|c|c|}
\hline \multirow{6}{*}{ (a) } & B & B & {$[1]$} & {$[0]$} & {$[0]$} & [1] & $\ldots$ & {$[1]$} & {$[0]$} & {$[0]$} & {$[1]$} & {$[1]$} & {$[0]$} \\
\hline & [1] & {$[0]$} & B & B & {$[0]$} & {$[1]$} & $\cdots$ & {$[1]$} & {$[0]$} & {$[0]$} & {$[1]$} & {$[1]$} & {$[0]$} \\
\hline & {$[1]$} & {$[0]$} & {$[0]$} & {$[1]$} & B & $\mathrm{B}$ & $\cdots$ & {$[1]$} & {$[0]$} & {$[0]$} & {$[1]$} & {$[1]$} & {$[0]$} \\
\hline & {$[1]$} & {$[0]$} & {$[0]$} & {$[1]$} & {$[1]$} & {$[0]$} & $\ldots$ & $\mathrm{B}$ & $\mathrm{B}$ & {$[0]$} & {$[1]$} & {$[1]$} & {$[0]$} \\
\hline & {$[1]$} & {$[0]$} & {$[0]$} & {$[1]$} & {$[1]$} & {$[0]$} & $\ldots$ & {$[0]$} & {$[1]$} & B & B & {$[1]$} & {$[0]$} \\
\hline & {$[1]$} & {$[0]$} & {$[0]$} & {$[1]$} & {$[1]$} & {$[0]$} & $\ldots$ & {$[0]$} & {$[1]$} & {$[1]$} & {$[0]$} & B & B \\
\hline \multirow{6}{*}{ (b) } & $\mathrm{B}_{1}$ & $\mathrm{~B}_{1}$ & $(0)$ & (1) & (1) & $(0)$ & & $(0)$ & (1) & (1) & (0) & $(0)$ & (1) \\
\hline & $(0)$ & (1) & $\mathrm{B}_{1}$ & $\mathrm{~B}_{1}$ & (1) & (0) & $\ldots$ & $(0)$ & (1) & (1) & (0) & (0) & (1) \\
\hline & (0) & (1) & (1) & $(0)$ & $\mathrm{B}_{1}$ & $\mathrm{~B}_{1}$ & $\ldots$ & $(0)$ & (1) & (1) & (0) & (0) & (1) \\
\hline & (0) & (1) & (1) & $(0)$ & (0) & (1) & $\ldots$ & $\mathrm{B}_{1}$ & $\mathrm{~B}_{1}$ & (1) & (0) & (0) & (1) \\
\hline & (0) & (1) & (1) & $(0)$ & (0) & (1) & $\cdots$ & (1) & (0) & $\mathrm{B}_{1}$ & $\mathrm{~B}_{1}$ & (0) & (1) \\
\hline & (0) & (1) & (1) & $(0)$ & (0) & (1) & $\ldots$ & (1) & (0) & (0) & (1) & $\mathrm{B}_{1}$ & $\mathrm{~B}_{1}$ \\
\hline
\end{tabular}

Figure 15: Matrix appearing in the proof of theorem 11

By substracting to each row in (b) the corresponding row in (a) and by resorting then the columns, the matrix in figure 16 is obtained. This matrix has complete range if $P$ is even and $n_{i} \geq 2$ for all $i$. Therefore, the inequality $F(x) \geq c_{0}$ is facet-inducing for $\operatorname{MGRP}\left(G_{\mathcal{C}}\right)$. Again, it is not difficult to see that, as in theorem 8, condition (L2) of lifting theorem 5 is satisfied and, hence, the inequality is also facet-inducing for $\operatorname{MGRP}(G)$.

\section{Other Path-Bridge inequalities}

Consider the vectors $x^{*}, x^{* *}$ described in figure 17 with $x_{e}^{*}=x_{e}^{* *}=1$ for the non required arcs (expressed in thin lines) and $x_{e}^{*}=x_{e}^{* *}=0$ for the required links (bold 


\begin{tabular}{|cccccc|cccccc|}
\hline $\mathrm{B}$ & {$[1]$} & {$[1]$} & $\cdots$ & {$[1]$} & {$[1]$} & $\mathrm{B}$ & {$[0]$} & {$[0]$} & $\cdots$ & {$[0]$} & {$[0]$} \\
{$[0]$} & $\mathrm{B}$ & {$[1]$} & $\cdots$ & {$[1]$} & {$[1]$} & {$[1]$} & $\mathrm{B}$ & {$[0]$} & $\ldots$ & {$[0]$} & {$[0]$} \\
{$[0]$} & {$[0]$} & $\mathrm{B}$ & $\ldots$ & {$[1]$} & {$[1]$} & {$[1]$} & {$[1]$} & $\mathrm{B}$ & $\ldots$ & {$[0]$} & {$[0]$} \\
& & & & & & & & & & & \\
& & & $\ddots$ & & & & & & $\ddots$ & & \\
{$[0]$} & {$[0]$} & {$[0]$} & $\cdots$ & $\mathrm{B}$ & {$[1]$} & {$[1]$} & {$[1]$} & {$[1]$} & $\cdots$ & $\mathrm{B}$ & {$[0]$} \\
& & & & & & & & & & & \\
{$[0]$} & {$[0]$} & {$[0]$} & $\cdots$ & {$[0]$} & $\mathrm{B}$ & {$[1]$} & {$[1]$} & {$[1]$} & $\cdots$ & {$[1]$} & $\mathrm{B}$ \\
& & & & & & & & & & & \\
\hline$(0)$ & $(-1)$ & $(-1)$ & $\cdots$ & $(-1)$ & $(-1)$ & $(0)$ & $(1)$ & $(1)$ & $\cdots$ & $(1)$ & $(1)$ \\
$(1)$ & $(0)$ & $(-1)$ & $\cdots$ & $(-1)$ & $(-1)$ & $(-1)$ & $(0)$ & $(1)$ & $\cdots$ & $(1)$ & $(1)$ \\
$(1)$ & $(1)$ & $(0)$ & $\cdots$ & $(-1)$ & $(-1)$ & $(-1)$ & $(-1)$ & $(0)$ & $\cdots$ & $(1)$ & $(1)$ \\
& & & $\ddots$ & & & & & & $\ddots$ & & \\
$(1)$ & $(1)$ & $(1)$ & $\cdots$ & $(0)$ & $(-1)$ & $(-1)$ & $(-1)$ & $(-1)$ & $\cdots$ & $(0)$ & $(1)$ \\
$(1)$ & $(1)$ & $(1)$ & $\cdots$ & $(1)$ & $(0)$ & $(-1)$ & $(-1)$ & $(-1)$ & $\cdots$ & $(-1)$ & $(0)$ \\
\hline
\end{tabular}

Figure 16: Matrix appearing in the proof of theorem 11

lines). Vectors $x^{*}+x^{R}$ and $x^{* *}+x^{R}$ are not tours for the MGRP although vectors $x^{*}$ and $x^{* *}$ can be obtained as vertices of the polyhedron defined by all previously described inequalities.
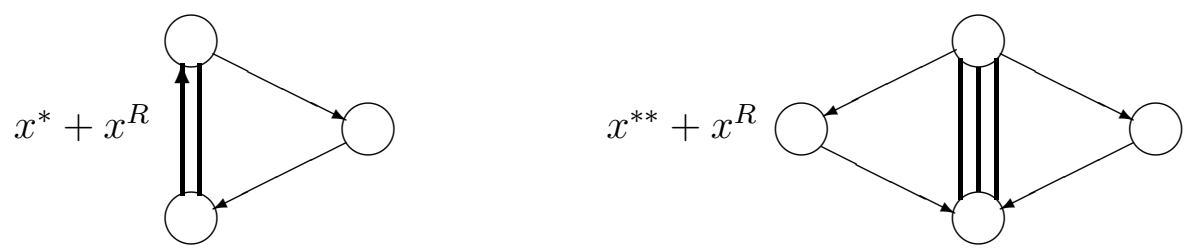

Figure 17: Two vectors satisfying all PB inequalities.

We next describe some new inequalities that 'cut-off' such 'solutions', which will be called $\mathrm{PB}_{02}$ inequalities.

Consider a $\mathrm{PB}$ configuration as in the previous section except that now $n_{i}$ can be equal to 1 in every path $i=1,2, \ldots, P$. Let us divide the set of paths into two types: paths of type $\mathcal{A Z}$ and paths of type $\mathcal{Z} \mathcal{A}$, and let us redefine the cost functions $c^{E}$ and $c^{A}$ as follows:

- For each path $i$ of type $\mathcal{A Z}$, we define: $c^{A}\left(M_{0}^{i}, M_{1}^{i}\right)=0$ and $c^{A}\left(M_{1}^{i}, M_{0}^{i}\right)=\frac{2}{n_{i}}$. The remaining arcs in the path have coefficient $\frac{1}{n_{i}}$ and, for the internal arcs, $c^{A}\left(M_{j}^{i}, M_{q}^{i}\right)$ is the cost of the shortest path from $M_{j}^{i}$ to $M_{q}^{i}$ using only external arcs. 
- For each path $i$ of type $\mathcal{Z} \mathcal{A}$, we define $c^{A}\left(M_{0}^{i}, M_{1}^{i}\right)=\frac{2}{n_{i}}$ and $c^{A}\left(M_{1}^{i}, M_{0}^{i}\right)=0$. The remaining arcs in the path have coefficient $\frac{1}{n_{i}}$. For each internal arc, $c^{A}\left(M_{j}^{i}, M_{q}^{i}\right)$ is the cost of the shortest path from $M_{j}^{i}$ to $M_{q}^{i}$ using only external arcs.

- Order the crossing arcs (if any) in an arbitrary way $a_{1}, a_{2}, \ldots, a_{h} \in \mathcal{C} r$. For $i=1$ to $h$ let $c_{a_{i}}$ be the maximum value such that $a_{i}$ belongs to a tour of $c$-length $P+1$ using only links from $\left(E_{\mathcal{C}} \cup A_{\mathcal{C}}\right) \backslash \mathcal{C} r$ and $\left\{a_{1}, \ldots, a_{i}\right\}$ (sequential lifting).

In other words,

$$
\begin{aligned}
& c^{A}(A, Z)=c^{A}(Z, A)=c^{E}(A, Z)=1 \\
& c^{A}\left(M_{j}^{i}, M_{q}^{i}\right)=\frac{|j-q|}{n_{i}}, \\
& c^{A}\left(M_{0}^{i}, M_{q}^{i}\right)=\frac{q-1}{n_{i}} \text { and } c^{A}\left(M_{q}^{i}, M_{0}^{i}\right)=\frac{q+1}{n_{i}} \\
& \text { if path } i \text { is of type } \mathcal{A \mathcal { Z } ,} \quad \forall q \in\left\{1,2, \ldots, n_{i}\right\} \\
& c^{A}\left(M_{0}^{i}, M_{q}^{i}\right)=\frac{q+1}{n_{i}} \text { and } c^{A}\left(M_{q}^{i}, M_{0}^{i}\right)=\frac{q-1}{n_{i}} \\
& \text { if path } i \text { is of type } \mathcal{Z} \mathcal{A}, \quad \forall q \in\left\{1,2, \ldots, n_{i}\right\}
\end{aligned}
$$

Such a configuration will be called $P B_{02}$ configuration (see figure 18).

Note that each path has a pair of opposite external arcs with coefficients 0 and $\frac{2}{n_{i}}$, while the other external edges have coefficient $\frac{1}{n_{i}}$. Furthermore, the direction of the arc with coefficient 0 determines whether the corresponding path is of type $\mathcal{A Z}$ or type $\mathcal{Z} \mathcal{A}$.

The $P B_{02}$ inequality corresponding to this new configuration is defined by:

$$
\sum_{e \in E \cup A} c_{e} x_{e} \geq P+1
$$

Again, we can define the special $n$-regular $P B_{02}$ inequality in which $n_{i}=n$ for each path $i$. In this case, by multiplying by $n$, the inequality (12) can be rewritten in the following way:

$$
\sum_{e \in E \cup A} c_{e} x_{e} \geq P n+n
$$

where

$$
\begin{aligned}
& c^{A}(A, Z)=c^{A}(Z, A)=c^{E}(A, Z)=n \\
& c^{A}\left(M_{j}^{i}, M_{q}^{i}\right)=|j-q|, \quad \forall j, q \in\left\{1,2, \ldots, n_{i}+1\right\} \\
& c^{A}\left(M_{0}^{i}, M_{q}^{i}\right)=q-1 \text { and } c^{A}\left(M_{q}^{i}, M_{0}^{i}\right)=q+1 \\
& \text { if path } i \text { is of type } \mathcal{A Z}, \quad \forall q \in\left\{1,2, \ldots, n_{i}\right\} \\
& c^{A}\left(M_{0}^{i}, M_{q}^{i}\right)=q+1 \text { and } c^{A}\left(M_{q}^{i}, M_{0}^{i}\right)=q-1 \\
& \text { if path } i \text { is of type } \mathcal{Z} \mathcal{A}, \quad \forall q \in\left\{1,2, \ldots, n_{i}\right\}
\end{aligned}
$$




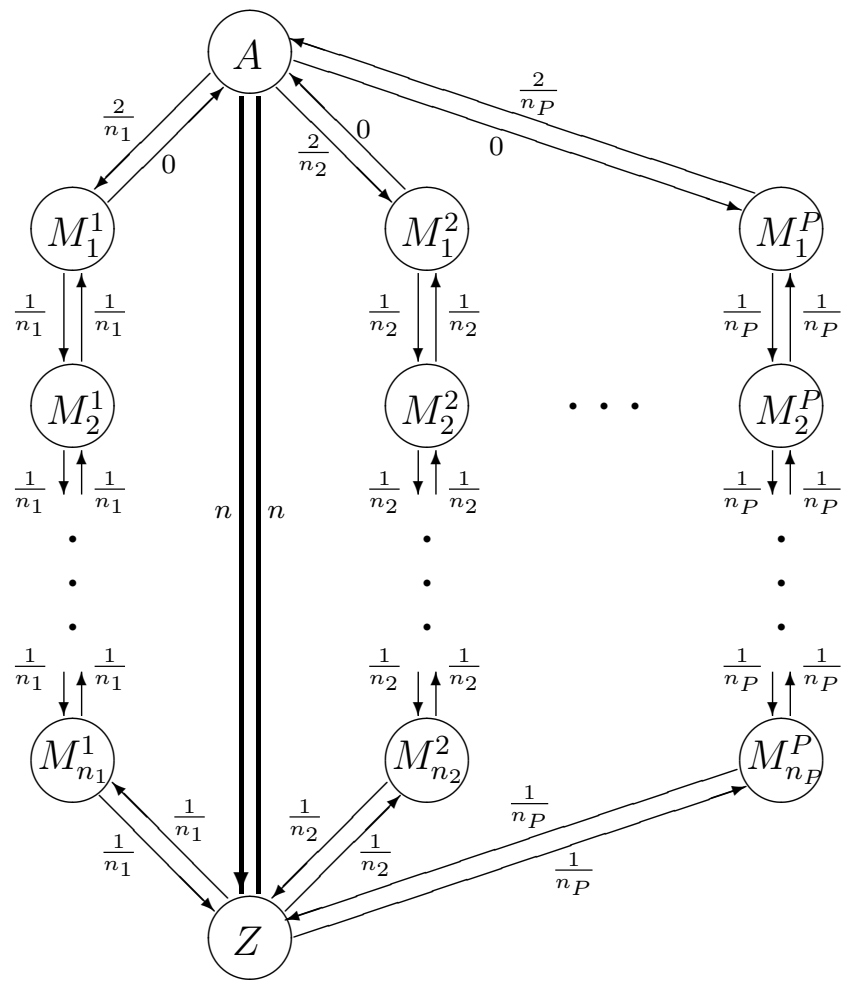

Figure 18: $\mathrm{PB}_{02}$ configuration.

Constraints (12) and (13) are called $\mathrm{PB}_{02}$ inequalities because they are similar to the standard $\mathrm{PB}$ inequalities, being the most relevant difference that a pair of external opposite arcs in each path have changed their coefficients to 0 and $\frac{2}{n_{i}}$.

Theorem $12 P_{02}$ inequalities are valid for $M G R P(G)$.

Proof: It suffices to prove validity in the configuration graph $G_{\mathcal{C}}$. Let $F(x) \geq P+1$ be a $\mathrm{PB}_{02}$ inequality. By the definition of the coefficients for internal and crossing arcs, it suffices to prove validity for each semitour $x$ for the MGRP on $G_{\mathcal{C}}$ using only links in $(A: Z) \cup \mathcal{E} x$.

For each path $i$, if there exists a $j^{*} \in\left\{0,1,2, \ldots, n_{i}\right\}$ such that $x\left(M_{j^{*}}^{i}: M_{j^{*}+1}^{i}\right)=$ $x\left(M_{j^{*}+1}^{i}: M_{j^{*}}^{i}\right)=0$, then connectivity and evenness conditions for the semitour $x$ in this path implies that $x\left(M_{j}^{i}: M_{j+1}^{i}\right) \geq 2$ for all $j=0,1, \ldots, j^{*}-1, j^{*}+1, \ldots, n_{i}$. The associated $c$-cost is, at least, 2 . Otherwise, the semitour uses either at least one copy of each arc in the path $A, M_{1}^{i}, M_{2}^{i}, \ldots, M_{n_{i}}^{i}, Z$ or one copy of each arc in the path $Z, M_{n_{i}}^{i}, \ldots, M_{1}^{i}, A$. In this case, the associated $c$-cost is, at least, 1 .

Given that in a $\mathrm{PB}_{02}$ configuration $P+B$ is odd, if a semitour $x$ traverses every path exactly once, it must traverse also a link in $(A: Z)$ (with coeficient 1 ) and, therefore, $F(x) \geq P+1$. Otherwise, it traverses, at least, a path twice or a path with a $j^{*}$ as defined above. In any case, $F(x) \geq 2+(P-1)=P+1$. 
Theorem $13 P B_{02}$ inequalities are facet-inducing for $M G R P(G)$ if

$\epsilon \geq|\alpha+A Z-\beta-Z A|+1$ where $\epsilon=|E(A: Z)|, \alpha=\left|A_{R}(A: Z)\right|, A Z$ is the number of paths of type $\mathcal{A Z}, \beta=\left|A_{R}(Z: A)\right|$ and $Z A$ is the number of paths of type $\mathcal{Z} \mathcal{A}$.

Proof: Let $F(x) \geq P+1$ denote the $\mathrm{PB}_{02}$ inequality. We will show first that $F(x) \geq c_{0}$ is facet-inducing for $\operatorname{MGRP}\left(G_{\mathcal{C}}\right)$. Given that $\epsilon \geq 1$,

$$
\begin{aligned}
\operatorname{dim}\left(\operatorname{MGRP}\left(G_{\mathcal{C}}\right)=\right. & |(A: Z) \cup \mathcal{I} n \cup \mathcal{C} r|+2 \sum\left(n_{i}+1\right)-\left(\sum n_{i}+1\right)+1= \\
& =|(A: Z) \cup \mathcal{I} n \cup \mathcal{C} r|+\sum\left(n_{i}+2\right)
\end{aligned}
$$

and this is the number of linearly independent semitours satisfying $F(x)=P+1$ we have to find.

As for the standard PB inequalities, for each link $e \in(A: Z) \cup \mathcal{I} n \cup \mathcal{C} r$, there always exists a semitour for the MGRP in $G_{\mathcal{C}}$ satisfying $F(x)=P+1$ and using $e$ exactly once and external arcs. Hence, only $\sum\left(n_{i}+2\right)$ more linearly independent semitours, using only external arcs, are needed.

(a) For each path $i^{*} \in\{1,2, \ldots, P\}$ and for each $j^{*} \in\left\{0,1,2, \ldots, n_{i^{*}}\right\}$, we consider the semitour that uses the two opposite arcs in each $\left(B_{j}^{i^{*}}: B_{j+1}^{i^{*}}\right)$, except for $j=j^{*}$, and one single arc in each $\left(B_{j}^{i}: B_{j+1}^{i}\right), \forall i \neq i^{*}$ (if any), in such a way that paths of type $\mathcal{A Z}$ are oriented from $A$ to $Z$ and paths of type $\mathcal{Z} \mathcal{A}$ are oriented from $Z$ to $A$. In other words, $P-1$ of the paths joining $A$ and $Z$ are used once, in the direction given by the arc with zero coefficient, while the last path is broken into two parts and each part is used twice. These sets of arcs are semitours for the MGRP on $G_{\mathcal{C}}$ because, given that $\epsilon \geq|\alpha+A Z-\beta-Z A|+1$, by adding to them the required links we obtain an eulerian graph. Furthermore, all these semitours satisfy $F(x)=P+1$, since the $P-1$ paths used once have a $c$-cost of 1 and the "broken" path used twice has a $c$-cost of 2 .

(b) For each path $i, i=1,2, \ldots, P$, we build a "special" semitour considering two copies of each arc in the path $i$, in the direction given by the arc with zero coefficient, and one copy of each arc in the remaining paths, always in the direction given by the arc with zero coefficient. Note that for this semitour $F(x)=P+1$, since arcs in the path $i$ have a total $c$-cost of 2 , while we obtain a total $c$-cost of $P-1$ for the remaining paths.

Therefore, we have built $n_{i}+2$ semitours for the MGRP, satisfying $F(x)=P+$ 1 , associated to each path $i$. In order to show that these $\sum\left(n_{i}+2\right)$ semitours are linearly independent, consider their incidence matrix relative to the external arcs. In this matrix, the columns correspond to the external arcs and are grouped by paths. Columns associated to arcs in each path are sorted in the following way. For the paths of type $\mathcal{A Z}$, the first half correspond to the arcs oriented from $A$ to $Z$ and the second one to those oriented from $Z$ to $A$, while for the paths of type $\mathcal{Z} \mathcal{A}$ the ordering is the opposite.

By expressing the semitours as rows of this matrix (sorted as (a) and (b) before), we obtain the matrix showed in figure 19. We have used the same notation as in the proof of theorem 11 .

By resorting the columns, the matrix in figure 20 is obtained. This matrix has complete range for $P \geq 1$ and $n_{i} \geq 1$ for all $i$. Therefore, the inequality $F(x) \geq P+1$ 
is facet-inducing for $\operatorname{MGRP}\left(G_{\mathcal{C}}\right)$. Again, as in theorem 8, condition (L2) of lifting theorem 5 is satisfied and therefore the inequality is also facet-inducing for $\operatorname{MGRP}(G)$.

\begin{tabular}{c|ccccccccc}
\hline & B & B & {$[1]$} & {$[0]$} & {$[1]$} & {$[0]$} & $\cdots$ & {$[1]$} & {$[0]$} \\
{$[1]$} & {$[0]$} & B & B & {$[1]$} & {$[0]$} & $\cdots$ & {$[1]$} & {$[0]$} \\
& {$[1]$} & {$[0]$} & {$[1]$} & {$[0]$} & B & B & $\cdots$ & {$[1]$} & {$[0]$} \\
(a) & & & & & & & & & \\
& & & & & & & $\ddots$ & & \\
& {$[1]$} & {$[0]$} & {$[1]$} & {$[0]$} & {$[1]$} & {$[0]$} & $\cdots$ & B & B \\
& & & & & & & & & \\
\hline & $(2)$ & $(0)$ & $(1)$ & $(0)$ & $(1)$ & $(0)$ & $\cdots$ & $(1)$ & $(0)$ \\
& $(1)$ & $(0)$ & $(2)$ & $(0)$ & $(1)$ & $(0)$ & $\cdots$ & $(1)$ & $(0)$ \\
& $(1)$ & $(0)$ & $(1)$ & $(0)$ & $(2)$ & $(0)$ & $\cdots$ & $(1)$ & $(0)$ \\
& $(1)$ & $(0)$ & $(1)$ & $(0)$ & $(1)$ & $(0)$ & $\cdots$ & $(2)$ & $(0)$ \\
\hline
\end{tabular}

Figure 19: Matrix appearing in the proof of theorem 13

\begin{tabular}{|cccccc|cccccc|}
\hline $\mathrm{B}$ & {$[0]$} & {$[0]$} & $\cdots$ & {$[0]$} & {$[0]$} & $\mathrm{B}$ & {$[1]$} & {$[1]$} & $\cdots$ & {$[1]$} & {$[1]$} \\
{$[0]$} & $\mathrm{B}$ & {$[0]$} & $\cdots$ & {$[0]$} & {$[0]$} & {$[1]$} & $\mathrm{B}$ & {$[1]$} & $\cdots$ & {$[1]$} & {$[1]$} \\
{$[0]$} & {$[0]$} & $\mathrm{B}$ & $\cdots$ & {$[0]$} & {$[0]$} & {$[1]$} & {$[1]$} & $\mathrm{B}$ & $\cdots$ & {$[1]$} & {$[1]$} \\
& & & & & & & & & & & \\
{$[0]$} & {$[0]$} & {$[0]$} & $\cdots$ & $\mathrm{B}$ & {$[0]$} & {$[1]$} & {$[1]$} & {$[1]$} & $\cdots$ & $\mathrm{B}$ & {$[1]$} \\
{$[0]$} & {$[0]$} & {$[0]$} & $\cdots$ & {$[0]$} & $\mathrm{B}$ & {$[1]$} & {$[1]$} & {$[1]$} & $\cdots$ & {$[1]$} & $\mathrm{B}$ \\
& & & & & & & & & & & \\
\hline$(0)$ & $(0)$ & $(0)$ & $\cdots$ & $(0)$ & $(0)$ & $(2)$ & $(1)$ & $(1)$ & $\cdots$ & $(1)$ & $(1)$ \\
$(0)$ & $(0)$ & $(0)$ & $\cdots$ & $(0)$ & $(0)$ & $(1)$ & $(2)$ & $(1)$ & $\cdots$ & $(1)$ & $(1)$ \\
$(0)$ & $(0)$ & $(0)$ & $\cdots$ & $(0)$ & $(0)$ & $(1)$ & $(1)$ & $(2)$ & $\cdots$ & $(1)$ & $(1)$ \\
$(0)$ & $(0)$ & $(0)$ & $\cdots$ & $(0)$ & $(0)$ & $(1)$ & $(1)$ & $(1)$ & $\cdots$ & $(2)$ & $(1)$ \\
$(0)$ & $(0)$ & $(0)$ & $\cdots$ & $(0)$ & $(0)$ & $(1)$ & $(1)$ & $(1)$ & $\cdots$ & $(1)$ & $(2)$ \\
\hline
\end{tabular}

Figure 20: Matrix appearing in the proof of theorem 13 
In the $\mathrm{PB}_{02}$ configuration, coefficients $(0,2)$ can be associated with any pair of opposite external $\operatorname{arcs}\left(M_{j}^{i}: M_{j+1}^{i}\right)$ in any path $i, i \in\{0,1,2, \ldots, P\}$. Nevertheless, all these inequalities induce the same facet of $\operatorname{MGRP}(G)$ (given the same ordering for the crossing edges in the sequential lifting process).

Standard $\mathrm{PB}$ and $\mathrm{PB}_{02}$ inequalities induce different facets of $\operatorname{MGRP}(G)$. Note that, unlike standard $\mathrm{PB}$ inequalities, $\mathrm{PB}_{02}$ inequalities also induce facets of $\operatorname{MGRP}(G)$ when $n_{i}=1$ for some path $i$.

For $P=1$, we obtain the $\mathrm{K}-\mathrm{C}_{02}$ configuration whose skeleton is showed in figure 21. Note that internal arcs $\left(M_{i}, M_{j}\right)$ (not represented in figure 21) have a cost equal to the length of the shortest path from $M_{i}$ to $M_{j}$ using external arcs. The corresponding $K-C_{02}$ inequality is:

$$
\sum_{e \in E \cup A} c_{e} x_{e} \geq 2(K-1)
$$

These inequalities cut-off the 'solution' $x^{*}$ presented in figure 17 , since the $\mathrm{K}-\mathrm{C}_{02}$ inequality is $F(x) \geq 2$ but $F\left(x^{*}\right)=1$.

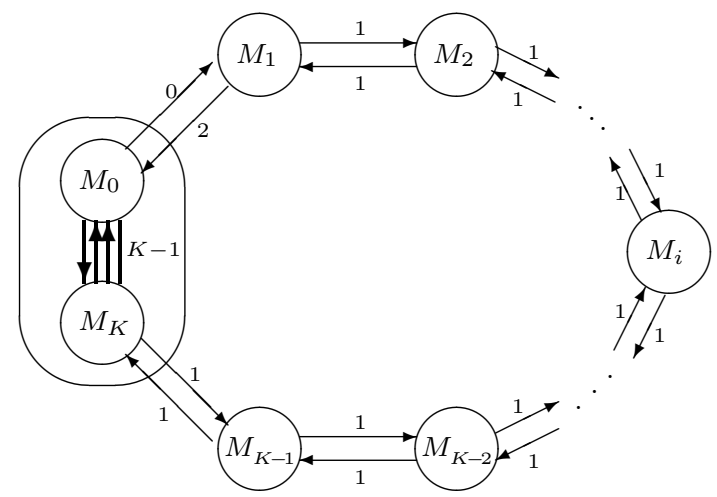

Figure 21: $\mathrm{K}-\mathrm{C}_{02}$ configuration.

In the definition of the $\mathrm{K}-\mathrm{C}_{02}$ configuration we have required the existence of two opposite arcs in each $\left(M_{i}: M_{i+1}\right)$, for $i=1,2, \ldots, K-1$. Nevertheless, in some cases this condition can be relaxed:

(a) For $K=2$, the three $\mathrm{K}-\mathrm{C}_{02}$ inequalities corresponding to the configurations of figure 22 are facet-inducing if $\epsilon \geq \max \{\alpha-\beta, \beta-\alpha+2\}$.

(b) For $K \geq 3$, if the subgraph induced by the links in $\mathcal{E} x \cup \mathcal{I}$ n is not strongly connected, i.e., there are no arcs from nodes $\left\{M_{j+1}, M_{j+2} \ldots, M_{K}\right\}$ to nodes $\left\{M_{0}, M_{1}, \ldots, M_{j}\right\}$ for some $j$, then the corresponding $\mathrm{K}-\mathrm{C}_{02}$ inequality can be shown to be dominated by the $2-\mathrm{C}_{02}$ inequality corresponding to the configuration with node sets: $M_{0}^{\prime}=\left\{M_{0}, M_{1}, \ldots, M_{j-1}\right\}, M_{1}^{\prime}=M_{j}$ and $M_{2}^{\prime}=\left\{M_{j+1}, M_{j+2} \ldots, M_{K}\right\}$.

(c) For $K \geq 3$, if the subgraph induced by the links in $\mathcal{E} x \cup \mathcal{I} n$ is strongly connected, the corresponding $\mathrm{K}-\mathrm{C}_{02}$ inequality can be, or not, facet-inducing. 

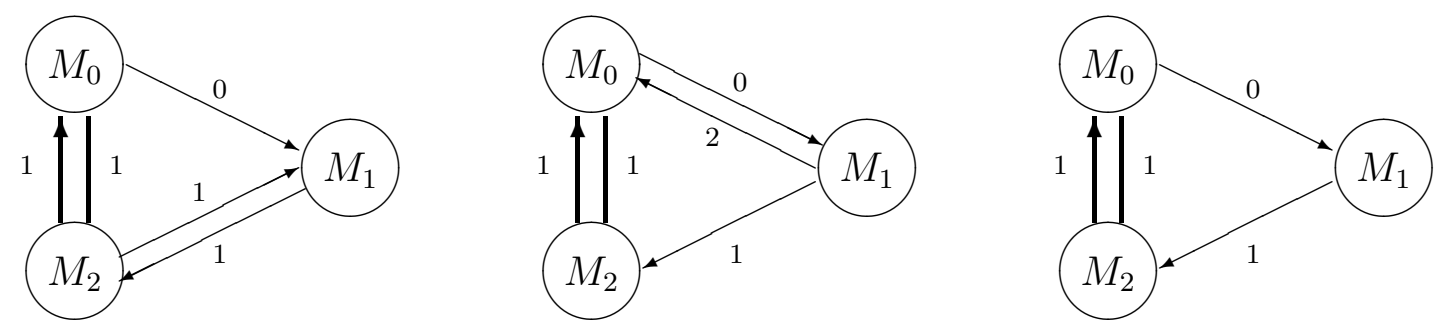

Figure 22: Other 2- $\mathrm{C}_{02}$ facet-inducing inequalities.

Finally, when $\epsilon=0$, although $\alpha=\beta$, the $\mathrm{K}-\mathrm{C}_{02}$ inequality can be shown to be dominated by the standard K-C inequality and, therefore, it is not facet-inducing.

If $P=2$ and $n_{i}=n$ for all $i$, we obtain the regular $2-\mathrm{PB}_{02}$ configuration. Figure 23 shows two skeletons (in the regular case) out of the 3 possible configurations that result from the assignment of the 2 paths to the types $\mathcal{A Z}$ and $\mathcal{Z} \mathcal{A}$. Again, internal arcs (not represented in the figure) have a cost equal to the length of the shortest path using external arcs. The corresponding regular 2- $P B_{02}$ inequality is:

$$
\sum_{e \in E \cup A} c_{e} x_{e} \geq 3 K
$$
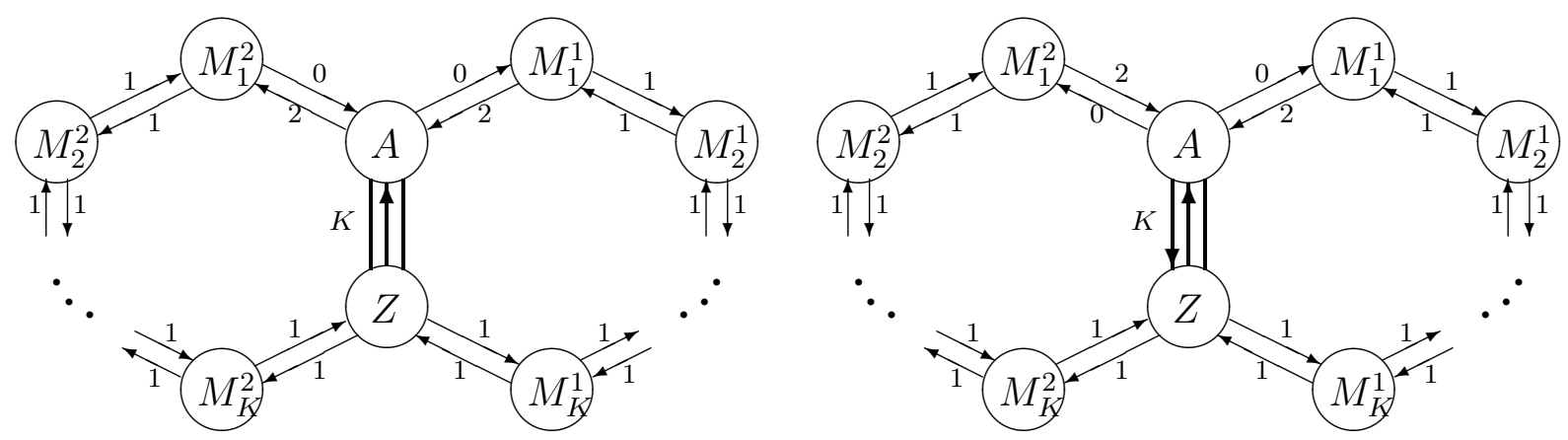

Figure 23: Two Regular 2- $\mathrm{PB}_{02}$ configurations.

A $2-\mathrm{PB}_{02}$ inequality cut-off the vector $x^{* *}$ in figure 17 , since the inequality is $F(x) \geq 3$ while $F\left(x^{* *}\right)=2$.

\section{Computational Results.}

We have devised a cutting-plane algorithm with separation procedures for the connectivity, $R$-odd cut and balanced-set inequalities. The initial LP relaxation includes the system equations (6), one connectivity inequality (4) for each $R$-set, one balanced-set inequality (5) for each 'unbalanced' vertex (i.e., the balanced-set inequality corresponding to each vertex $i \in V$ such that $|E(i)|<\left|A_{R}^{+}(i)\right|-\left|A_{R}^{-}(i)\right|$ 
or the balanced-set inequality corresponding to $V \backslash\{i\}$ for each vertex $i \in V$ such that $\left.|E(i)|<\left|A_{R}^{-}(i)\right|-\left|A_{R}^{+}(i)\right|\right)$, and one $R$-odd cut inequality (8) for each $R$-odd 'balanced' vertex.

The separation problems associated with connectivity and $R$-odd cut inequalities are solvable in polynomial time by means of max-flow calculations and the Padberg \& Rao (1982) procedure to find minimum odd cut-sets. Standard heuristics for both problems were also implemented. Nobert \& Picard (1996) showed that identifying violated balanced-set inequalities for the MCPP can also be solved in polynomial time (see Benavent, Corberán and Sanchis, 2000, for a short and direct proof of this important result). This result is easily extended to the MGRP. In the course of the algorithm, additional inequalities of the above mentioned classes are generated as they are found to be violated. When this is no longer possible and the LP solution is still not integral, we invoke branch and bound. If the resulting integer solution is feasible for the MGRP, it is optimal. Otherwise, the procedure terminates with a tight lower bound, but no feasible MGRP solution.

This algorithm has been coded in $C$ and run on a Personal Computer with a $400 \mathrm{MHz}$ Pentium II processor. It is a rough algorithm implemented to provide lower bounds to a set of $100 \mathrm{MRPP}$ instances randomly generated for testing the constructive and the tabu search algorithms described in Corberán, Martí \& Romero (2000).

The graph generator works as follows: a given number of vertices, $|V|$, is equally distributed among the desired number $p$ of $R$-sets. Initially, each $R$-set has $\left\lfloor\frac{|V|}{p}\right\rfloor$ vertices, except possibly the last one which also has the difference up to $|V|$. Arcs and edges are generated, up to a prefixed number, by randomly selecting two vertices and adding the corresponding link. To generate required links, if the two vertices do not belong to the same $R$-set they are rejected and a new selection is made. Note that, as the required links may not connect all the vertices in a $R$-set, the final number of $R$-sets may be diferent to the one initially desired. Furthermore, the final number of non-required arcs might be larger, in some instances, than the prefixed number $\left|A \backslash A_{R}\right|$, in order to obtain a strongly connected graph. This generator allows up to four links between two vertices: one for each kind of link (required and non-required arc, required and non-required edge). We have randomly generated 100 instances with the following ranges: $3 \leq p \leq 12,20 \leq|V| \leq 100,15 \leq|E| \leq 220,5 \leq\left|E_{R}\right| \leq 150,50 \leq|A| \leq 350$, $5 \leq\left|A_{R}\right| \leq 200$. The cost of each link has been randomly generated in the range $1-20$.

Tables 1, 2 and 3 present the characteristics of the instances, the lower bound obtained with our cutting-plane procedure (column LB), the lower bound obtained with the branch and bound (column $\mathrm{B} \& \mathrm{~B}$ ) and the number of nodes of the $\mathrm{B} \& \mathrm{~B}$ tree (column $\mathrm{Nd}$ ), the best upper bound obtained with the mentioned heuristics (column UB) and the CPU time in seconds (column Time). An entry marked with an asterisk means that an optimal solution is reached. Note that the cutting-plane procedure is capable of finding an optimal solution in 71 out of the 100 MRPP instances and 
that this number increases up to 93 after invoking branch and bound. Although general MGRP instances will be harder to solve, we consider that these are encouraging results in order to implement a better cutting-plane or branch and cut algorithm.

\section{Relationship with other Routing Problems.}

In previous sections we have studied the polyhedron associated with the MGRP. We now present the application of our results to other known routing problems: the Mixed Chinese Postman Problem, the Undirected, Directed and Mixed Rural Postman Problems and the Graphical Asymmetric Traveling Salesman Problem.

The conclusions are conditioned by the fact that the original graph is transformed into a new simplified graph without non-required edges (they have been replaced by a pair of opposite non-required arcs). Hence, the results obtained so far for the MGRP can only be applied to instances and Problems without non-required edges.

\section{Mixed CPP:}

This problem is a particular case of the MGRP and arises when all the links of the graph are required to be traversed. The approach described in Nobert \& Picard (1996) uses trivial inequalities (1), balanced-set inequalities (5) and $R$-odd cut inequalities (8) to design an algorithm which has proven to be highly effective. However, the issue of whether these inequalities induce or not facets of the associated polyhedron has not been studied. Given an instance $G=(V, E \cup A)$ of MCPP, our results for MGRP imply that $\operatorname{MCPP}(G)$ is a polyhedron of dimension $|E \cup A|-q+1$, where again $q$ is the number of connected components of graph $(V, E)$, and the above inequalities $(1),(5)$ and (8) are facet-inducing under the conditions described in their respective sections.

\section{Undirected RPP:}

Results obtained for the Mixed GRP cannot be directly applied to the Undirected RPP due to the existence of non-required edges. To study the MGRP without replacing the non required edges by two arcs may be more attractive from a theoretical point of view, but it is also quite more difficult. Consider, for example, a K-C configuration in which there may exist, or not, edges between any pair of nodes $M_{i}, M_{j}$. In this case, the dimension of $\operatorname{MGRP}\left(G_{\mathcal{C}}\right)$ depends on the number and the disposal of such edges.

\section{Directed RPP:}

Given that this problem is defined on directed graphs, all the results obtained for the MGRP not implied by the existence of required edges are also valid for the DRPP. Indeed, given a (simplified) instance $G=\left(V_{R}, A_{R} \cup A_{N R}\right)$ of DRPP, we have that $\operatorname{DRPP}(G)$ is a polyhedron of dimension $\left|A_{R} \cup A_{N R}\right|-|V|+1$, and the following inequalities are facet-inducing:

- Trivial inequalities: $\quad x_{a} \geq 0$ if $a \in A_{R} \cup A_{N R}$ is not a cut-arc. 
- Connectivity inequalities: $x\left(A^{+}(S)\right) \geq 1$, where $S \subset V$ is the union of $R$-sets, if $G(S)$ and $G(V \backslash S)$ are strongly connected.

- Standard K-C inequalities if $\alpha=\beta$ (see section 5).

$\mathrm{K}-\mathrm{C}_{02}$ inequalities described in section 5 and $\mathrm{PB}$ inequalities described in section 5 are also valid for $\operatorname{DRPP}(G)$, but it is not possible to directly conclude if they are or not facet-inducing.

\section{Asymmetric GTSP:}

As it was mentioned before, GATSP is a special case of MGRP when $E=A_{R}=\emptyset$. Therefore, the results obtained in previous sections not involving required links also apply to GATSP. Hence, for example, $\operatorname{GATSP}(G)$ is an unbounded and non full-dimensional polyhedron, trivial inequalities are facet-inducing, all the remaining facet-inducing inequalities are configuration inequalities, connectivity inequalities and standard PB inequalities with $(A: Z)_{R}=\emptyset$ are facet inducing. These last inequalities correspond to the known s-path, s-wheelbarrow and s-bicycle inequalities. All these results were previously obtained by Chopra and Rinaldi (1996).

Conversely, it is worthwhile to point out that every non trivial valid (or facetinducing) inequality for the GATSP polyhedron provides a valid (or facet-inducing) inequality for $\operatorname{MGRP}(G)$. Given an instance $G=\left(V, E \cup A_{R} \cup A_{N R}\right)$ of MGRP, if we shrink the $R$-sets into a single node each and we remove the loops, we obtain an instance for the GATSP. This is also true if we shrink, into a single node, every vertex set $B_{j}$ in any partition $\mathcal{B}=\left\{B_{1}, B_{2}, \ldots, B_{r}\right\}$ of $V$ satisfying that each $R$-set $V^{i}$ is contained into one set $B_{j}$. Let us call GATSP configuration to any configuration $\mathcal{C}=\left(\mathcal{B}, c^{E}, c^{A}\right)$ on $G$ where $\mathcal{B}=\left\{B_{1}, B_{2}, \ldots, B_{r}\right\}$ satisfies the previous condition.

Theorem 14 Let $\mathcal{C}$ be a GATSP configuration on $G$ and let $G_{\mathcal{C}}$ be its corresponding configuration graph. If the configuration inequality associated with $G_{\mathcal{C}}$ is facet-inducing for $\operatorname{GATSP}\left(G_{\mathcal{C}}\right)$, then the configuration inequality associated with $G$ is facet-inducing for $M G R P(G)$.

Proof: Given that the graph $G_{\mathcal{C}}$ has no required links, $\operatorname{GATSP}\left(G_{\mathcal{C}}\right)=\operatorname{MGRP}\left(G_{\mathcal{C}}\right)$ and, hence, the inequality associated with $G_{\mathcal{C}}$ is facet-inducing for $\operatorname{MGRP}\left(G_{\mathcal{C}}\right)$. Furthemore, given that $G_{\mathcal{C}}$ is a directed graph, condition $(L 1)$ in the first lifting theorem 4 is obviously satisfied. Therefore, the configuration inequality associated with $G$ is facet-inducing for $\operatorname{MGRP}(G)$.

\section{Conclusions}

In this paper we have studied the polyhedron associated with the General Routing Problem on a mixed graph. To the best of our knowledge, this is the first polyhedral study of a routing problem defined on a mixed graph. MGRP generalizes several important and difficult Arc and Node Routing Problems as CPP, RPP and GTSP 
either defined on an undirected, directed or mixed graph.

We have defined several families of inequalities and shown that they define facets for the MGRP polyhedron. Some of these families are derived from facet-inducing inequalities for other routing problems polyhedra: connectivity, $R$-odd cut, standard K-C and standard PB inequalities for the undirected RPP and GRP (Corberán \& Sanchis 1994, 1998, Letchford 1997, 1999), balanced-set inequalities for the MCPP (Nobert \& Picard 1996) and all the known families for the GATSP (Chopra \& Rinaldi, 1996).

Some computational results obtained with a preliminary cutting-plane algorithm based on some of these inequalities are also given. We consider that these results show that the polyhedral description obtained in this paper can be useful when solving MGRP instances.

\section{Acknowledgements}

The authors would like to thank three anonymous referees for their helpful comments and suggestions that have greatly improved the contents and presentation of the paper.

\section{References}

[1] A.A. Assad \& B.L. Golden (1995): "Arc Routing Methods and Applications". In M.O. Ball, T.L. Magnanti, C.L. Monma \& G.L. Nemhauser (Eds.) Network Routing. Handbooks of Operations Research and Management Science, 8. Amsterdam: North Holland.

[2] E. Benavent, A. Corberán \& J.M. Sanchis (2000): "Linear Programming Based Methods for Solving Arc Routing Problems". In M. Dror (Ed.) Arc Routing: Theory, Solutions and Applications. Kluwer Academic Publishers.

[3] S. Chopra \& G. Rinaldi (1996): "The Graphical Asymmetric Traveling Salesman Polyhedron: Symmetric Inequalities". SIAM J. Discrete Math., 9, 4, 602-624.

[4] N. Christofides, E. Benavent, V. Campos, A. Corberán \& E. Mota (1984): “An optimal method for the Mixed Postman Problem". In P. Thoft-Christensen (Ed.) System Modelling and Optimization. Lecture Notes in Control and Information Sciences, 59. Berlin: Springer-Verlag.

[5] N. Christofides, V. Campos, A. Corberán \& E. Mota (1981): "An Algorithm for the Rural Postman Problem". Report IC.O.R.81.5, Imperial College, London.

[6] N. Christofides, V. Campos, A. Corberán \& E. Mota (1986): "An Algorithm for the Rural Postman Problem on a directed graph". Mathematical Programming Study 26, 155-166. 
[7] A. Corberán, R. Martí \& A. Romero (2000): "Heuristics for the Mixed Rural Postman Problem". Computers and Ops. Res. 27, 183-203.

[8] A. Corberán \& J.M. Sanchis (1994): "A Polyhedral Approach to the Rural Postman Problem". European Journal of Operational Research, 79, 95-114.

[9] A. Corberán \& J.M. Sanchis (1998): "The General Routing Problem Polyhedron: facets from RPP and GTSP Polyhedra". European Journal of Operational Research, 108, 538-550.

[10] G. Cornuèjols, J. Fonlupt \& D. Naddef (1985): "The Traveling Salesman Problem on a graph and some related integer polyhedra". Mathematical Programming, 33, $1-27$.

[11] M. Dror (Ed.) (2000): Arc Routing: Theory, Solutions and Applications. Kluwer Academic Publishers.

[12] J. Edmonds \& E. L. Johnson (1973): "Matching, Euler Tours and the Chinese Postman". Mathematical Programming, 5, 88-124.

[13] H.A. Eiselt, M. Gendreau \& G. Laporte (1995a): "Arc-Routing Problems, Part 1: the Chinese Postman Problem". Operations Research, 43, 231-242.

[14] H.A. Eiselt, M. Gendreau \& G. Laporte (1995b): "Arc-Routing Problems, Part 2: the Rural Postman Problem". Operations Research, 43, 399-414.

[15] L.R. Ford \& D.R. Fulkerson (1962) Flows in Networks. Princeton University Press, Princeton, NJ.

[16] M. Grötschel \& Z. Win (1992): "A cutting plane algorithm for the Windy Postman Problem". Mathematical Programming, 55, 339-358.

[17] J. Lenstra \& A. Rinnooy-Kan (1976): "On General Routing Problems". Networks, 6, 273-280.

[18] A.N. Letchford (1997): "New inequalities for the General Routing Problem". European Journal of Operational Research, 96, 317-322.

[19] A.N. Letchford (1999): "The General Routing Polyhedron: A Unifying Framework". European Journal of Operational Research, 112, 122-133.

[20] D. Naddef \& G. Rinaldi (1991): "The Symmetric Traveling Salesman Polytope and its graphical relaxation: Composition of valid inequalities". Mathematical Programming, 51, 359-400.

[21] Y. Nobert \& J.C. Picard (1996): "An optimal algorithm for the Mixed Chinese Postman Problem". Networks, 27, 95-108.

[22] M.W. Padberg \& M.R. Rao (1982): "Odd minimum cut-sets and b-matchings". Mathematics for Operations Research, 7, 67-80. 
[23] C.H. Papadimitriou (1976): "On the complexity of edge traversing". J. of the A.C.M., 23, 544-554. 


\begin{tabular}{|c|c|c|c|c|c|c|c|c|c|c|c|}
\hline & $p$ & $\mid \overline{|V|}$ & $|E|$ & $\left|E_{R}\right|$ & $|A|$ & $\left|A_{R}\right|$ & LB & $\mathrm{B} \& \mathrm{~B}$ & $\mathrm{Nd}$ & UB & Time \\
\hline g3c1 & 3 & 20 & 50 & 10 & 80 & 30 & $464^{*}$ & & & & 0,98 \\
\hline g3c2 & 3 & 30 & 140 & 40 & 200 & 50 & $946^{*}$ & & & & 2,58 \\
\hline g3c3 & 3 & 50 & 90 & 60 & 250 & 150 & $2375^{*}$ & & & & 20,43 \\
\hline g3c4 & 3 & 50 & 140 & 70 & 190 & 100 & $1697^{*}$ & & & & 4,73 \\
\hline g3c5 & 3 & 60 & 140 & 90 & 240 & 80 & $1791^{*}$ & & & & 5,71 \\
\hline g3c6 & 3 & 70 & 180 & 100 & 120 & 50 & $1663^{*}$ & & & & 4,23 \\
\hline g3c7 & 3 & 80 & 160 & 100 & 300 & 100 & 2320 & $2321^{*}$ & 13 & & 22,97 \\
\hline g3c8 & 5 & 90 & 130 & 40 & 200 & 50 & $1244^{*}$ & & & & 6,48 \\
\hline g3c9 & 3 & 100 & 220 & 150 & 300 & 200 & $4092^{*}$ & & & & 33,23 \\
\hline g3c10 & 3 & 100 & 200 & 130 & 250 & 150 & 3287 & $3288^{*}$ & 85 & & 49,57 \\
\hline $\mathrm{g} 4 \mathrm{c} 1$ & 4 & 20 & 105 & 25 & 80 & 50 & 793 & $793^{*}$ & 2 & & 4,12 \\
\hline $\mathrm{g} 4 \mathrm{c} 2$ & 3 & 40 & 140 & 20 & 150 & 100 & $1480^{*}$ & & & & 3,13 \\
\hline g4c3 & 4 & 50 & 60 & 40 & 250 & 150 & $2310^{*}$ & & & & 9,72 \\
\hline $\mathrm{g} 4 \mathrm{c} 4$ & 4 & 50 & 110 & 80 & 250 & 100 & $2079^{*}$ & & & & 9,99 \\
\hline g4c5 & 4 & 60 & 100 & 50 & 220 & 120 & $1852^{*}$ & & & & 3.57 \\
\hline g4c6 & 5 & 70 & 100 & 30 & 200 & 50 & $1010^{*}$ & & & & 3,25 \\
\hline $\mathrm{g} 4 \mathrm{c} 7$ & 4 & 80 & 190 & 90 & 350 & 50 & $1581^{*}$ & & & & 4,66 \\
\hline g4c8 & 3 & 90 & 150 & 100 & 320 & 200 & $3395^{*}$ & & & & 28,62 \\
\hline $\mathrm{g} 4 \mathrm{c} 9$ & 15 & 100 & 150 & 50 & 340 & 40 & $1186^{*}$ & & & & 5,33 \\
\hline $\mathrm{g} 4 \mathrm{c} 10$ & 2 & 100 & 150 & 100 & 220 & 130 & $2919^{*}$ & & & & 28,62 \\
\hline g5c1 & 5 & 20 & 50 & 20 & 80 & 10 & $369^{*}$ & & & & 2,25 \\
\hline $\mathrm{g} 5 \mathrm{c} 2$ & 5 & 40 & 90 & 50 & 150 & 60 & $1194^{*}$ & & & & 3,95 \\
\hline g5c3 & 5 & 50 & 180 & 30 & 250 & 100 & $1388^{*}$ & & & & 3,79 \\
\hline g5c4 & 4 & 50 & 140 & 40 & 210 & 60 & $1070^{*}$ & & & & 5,28 \\
\hline g5c5 & 6 & 60 & 110 & 30 & 250 & 50 & $1104^{*}$ & & & & 3,89 \\
\hline g5c6 & 5 & 70 & 160 & 100 & 270 & 200 & 3416 & $3416^{*}$ & 1 & & 17,53 \\
\hline g5c7 & 5 & 80 & 170 & 90 & 190 & 100 & 2337,75 & $2338^{*}$ & 1 & & 11,92 \\
\hline g5c8 & 5 & 90 & 180 & 80 & 300 & 150 & 2708 & $2708^{*}$ & 1 & & 20,21 \\
\hline g5c9 & 4 & 100 & 130 & 60 & 350 & 200 & $3118^{*}$ & & & & 10,27 \\
\hline g5c10 & 4 & 100 & 110 & 60 & 250 & 150 & $2796^{*}$ & & & & 8,79 \\
\hline g6c1 & 6 & 20 & 60 & 10 & 105 & 15 & $324^{*}$ & & & & 2,96 \\
\hline g6c2 & 6 & 40 & 100 & 30 & 180 & 80 & 1203,5 & $1204^{*}$ & 1 & & 4,44 \\
\hline g6c3 & 5 & 50 & 110 & 20 & 270 & 70 & $1106^{*}$ & & & & 3,96 \\
\hline g6c4 & 6 & 50 & 160 & 40 & 170 & 100 & $1667^{*}$ & & & & 4,12 \\
\hline g6c5 & 6 & 60 & 110 & 80 & 270 & 150 & $2545^{*}$ & & & & 5,33 \\
\hline g6c6 & 6 & 70 & 130 & 30 & 270 & 70 & $1259^{*}$ & & & & 2,85 \\
\hline g6c7 & 6 & 80 & 160 & 90 & 170 & 50 & $1679^{*}$ & & & & 5,49 \\
\hline g6c8 & 5 & 90 & 150 & 100 & 170 & 70 & $1986^{*}$ & & & & 13,02 \\
\hline g6c9 & 2 & 100 & 140 & 50 & 230 & 150 & $2750^{*}$ & & & & 10,71 \\
\hline g6c10 & 6 & 100 & 180 & 120 & 220 & 130 & $2952^{*}$ & & & & 28,90 \\
\hline
\end{tabular}

Table 1: Computational Results 1. 


\begin{tabular}{|c|c|c|c|c|c|c|c|c|c|c|c|}
\hline & $\overline{p p}$ & $\overline{|V|}$ & $|E|$ & $\mid \overline{\left|E_{R}\right|}$ & $|A|$ & $\mid \overline{\left|A_{R}\right|}$ & $\overline{\mathrm{LB}}$ & $\bar{B}$ B\&B & $\mathrm{Nd}$ & $\overline{\mathrm{UB}}$ & Time \\
\hline g7c1 & 6 & 20 & 55 & 5 & 130 & 10 & 235,5 & $236^{*}$ & 9 & & 1,87 \\
\hline g7c2 & 6 & 40 & 140 & 20 & 100 & 50 & $816^{*}$ & & & & 8,46 \\
\hline g7c3 & 8 & 50 & 140 & 50 & 280 & 30 & $847^{*}$ & & & & 5,93 \\
\hline g7c4 & 7 & 50 & 150 & 90 & 170 & 100 & 2043 & $2044^{*}$ & 3 & & 7,69 \\
\hline g7c5 & 7 & 60 & 170 & 40 & 240 & 150 & $2198^{*}$ & & & & 6,37 \\
\hline g7c6 & 7 & 70 & 150 & 50 & 300 & 200 & 2867,5 & $2868^{*}$ & 25 & & 12,04 \\
\hline g7c7 & 7 & 80 & 170 & 100 & 210 & 130 & 2642,5 & 2643 & 1 & 2650 & 9,39 \\
\hline g7c8 & 6 & 90 & 170 & 80 & 270 & 150 & 2630 & 2632 & 26 & 2634 & 8,91 \\
\hline g7c9 & 8 & 100 & 150 & 100 & 260 & 60 & $1902^{*}$ & & & & 19,44 \\
\hline g7c10 & 6 & 100 & 200 & 120 & 220 & 150 & $3024^{*}$ & & & & 16,48 \\
\hline g8c1 & 8 & 30 & 70 & 20 & 120 & 30 & 650,5 & $651^{*}$ & 1 & & 4,18 \\
\hline g8c2 & 8 & 40 & 90 & 20 & 190 & 90 & $1192^{*}$ & & & & 4,45 \\
\hline g8c3 & 7 & 50 & 50 & 30 & 360 & 60 & $1035^{*}$ & & & & 5,44 \\
\hline g8c4 & 8 & 50 & 120 & 70 & 220 & 120 & $1969^{*}$ & & & & 3,74 \\
\hline g8c5 & 8 & 60 & 120 & 40 & 180 & 100 & $1815^{*}$ & & & & 7,58 \\
\hline g8c6 & 8 & 70 & 150 & 90 & 230 & 70 & 1678,67 & $1679^{*}$ & 12 & & 9,84 \\
\hline g8c7 & 6 & 80 & 160 & 50 & 240 & 150 & $2620^{*}$ & & & & 23,07 \\
\hline g8c8 & 8 & 90 & 220 & 100 & 160 & 90 & $2213^{*}$ & & & & 14,67 \\
\hline g8c9 & 7 & 100 & 150 & 60 & 230 & 100 & $2365^{*}$ & & & & 23,67 \\
\hline g8c10 & 7 & 100 & 190 & 120 & 300 & 150 & $2974^{*}$ & & & & 14,11 \\
\hline g9c1 & 8 & 30 & 45 & 15 & 95 & 15 & $372^{*}$ & & & & 3,63 \\
\hline $\mathrm{g} 9 \mathrm{c} 2$ & 9 & 40 & 80 & 30 & 210 & 60 & $1025^{*}$ & & & & 4,67 \\
\hline g9c3 & 9 & 50 & 90 & 60 & 270 & 70 & $1537^{*}$ & & & & 6,98 \\
\hline $\mathrm{g} 9 \mathrm{c} 4$ & 9 & 50 & 80 & 40 & 150 & 50 & 966 & $967^{*}$ & 10 & & 4,56 \\
\hline g9c5 & 9 & 60 & 150 & 50 & 270 & 150 & $2444^{*}$ & & & & 6,04 \\
\hline g9c6 & 8 & 70 & 150 & 100 & 250 & 150 & $2915^{*}$ & & & & 5,39 \\
\hline g9c7 & 8 & 80 & 210 & 120 & 200 & 50 & 1994 & $1994^{*}$ & 1 & & 7,69 \\
\hline g9c8 & 7 & 90 & 150 & 50 & 200 & 120 & $2136^{*}$ & & & & 11,09 \\
\hline g9c9 & 7 & 100 & 130 & 70 & 290 & 200 & $3388^{*}$ & & & & 15,44 \\
\hline g9c10 & 7 & 100 & 150 & 100 & 240 & 70 & 1939 & $1940^{*}$ & 2 & & 16,59 \\
\hline g10c1 & 10 & 30 & 20 & 10 & 180 & 30 & 447 & 449 & 1 & 452 & 5,11 \\
\hline g10c2 & 10 & 40 & 40 & 20 & 170 & 80 & $1153^{*}$ & & & & 3,08 \\
\hline g10c3 & 10 & 50 & 80 & 50 & 290 & 40 & 950,5 & $951^{*}$ & 12 & & 3,69 \\
\hline g10c4 & 10 & 50 & 80 & 40 & 150 & 50 & $1157^{*}$ & & & & 5,16 \\
\hline g10c5 & 10 & 60 & 160 & 40 & 280 & 200 & $2633^{*}$ & & & & 7,30 \\
\hline g10c6 & 10 & 70 & 160 & 80 & 300 & 130 & 2162 & $2163^{*}$ & 24 & & 6,28 \\
\hline g10c7 & 10 & 80 & 190 & 130 & 270 & 150 & 3055,17 & 3058 & 19 & 3072 & 13,19 \\
\hline g10c8 & 9 & 90 & 150 & 100 & 250 & 160 & $2596^{*}$ & & & & 6,81 \\
\hline g10c9 & 8 & 100 & 160 & 70 & 210 & 100 & $2119^{*}$ & & & & 6,04 \\
\hline g10c10 & 10 & 100 & 210 & 150 & 200 & 70 & 2504,33 & $2505^{*}$ & 39 & & 8,19 \\
\hline
\end{tabular}

Table 2: Computational Results 2. 


\begin{tabular}{||c|c|c|c|c|c|c|c|c|c|r|r||}
\hline \hline & $p$ & $|V|$ & $|E|$ & $\left|E_{R}\right|$ & $|A|$ & $\left|A_{R}\right|$ & LB & B\&B & Nd & UB & Time \\
\hline g11c1 & 3 & 30 & 15 & 5 & 105 & 5 & $187^{*}$ & & & & 1,38 \\
\hline g11c2 & 9 & 40 & 35 & 15 & 220 & 20 & $505^{*}$ & & & & 4,06 \\
\hline g11c3 & 12 & 50 & 210 & 10 & 80 & 30 & $501^{*}$ & & & & 2,59 \\
\hline g11c4 & 10 & 50 & 80 & 40 & 150 & 50 & 1146 & $1146^{*}$ & 1 & & 4,89 \\
\hline g11c5 & 11 & 60 & 150 & 50 & 210 & 120 & $2029^{*}$ & & & & 5,05 \\
\hline g11c6 & 11 & 70 & 170 & 90 & 240 & 150 & 2625 & 2626 & 1 & 2642 & 5,60 \\
\hline g11c7 & 11 & 80 & 170 & 100 & 250 & 150 & $2926^{*}$ & & & & 5,38 \\
\hline g11c8 & 10 & 90 & 170 & 50 & 240 & 150 & $2395^{*}$ & & & & 4,29 \\
\hline g11c9 & 10 & 100 & 180 & 120 & 190 & 70 & 2166 & $2166^{*}$ & 1 & & 5,11 \\
\hline g11c10 & 10 & 100 & 170 & 70 & 220 & 100 & $2128^{*}$ & & & & 9,28 \\
\hline g12c1 & 7 & 30 & 105 & 5 & 55 & 5 & $131^{*}$ & & & & 1,92 \\
\hline g12c2 & 11 & 40 & 60 & 10 & 160 & 10 & $308^{*}$ & & & & 2,03 \\
\hline g12c3 & 15 & 50 & 25 & 15 & 320 & 20 & 480 & $480^{*}$ & 5 & & 2,69 \\
\hline g12c4 & 12 & 50 & 160 & 70 & 190 & 110 & 2053,87 & $2055^{*}$ & 22 & & 7,59 \\
\hline g12c5 & 12 & 60 & 160 & 40 & 170 & 70 & $1309^{*}$ & & & & 3,24 \\
\hline g12c6 & 12 & 70 & 180 & 80 & 210 & 120 & 2231 & 2232 & 42 & 2248 & 7,10 \\
\hline g12c7 & 12 & 80 & 180 & 110 & 220 & 90 & $2252^{*}$ & & & & 6,81 \\
\hline g12c8 & 11 & 90 & 170 & 60 & 250 & 150 & $2498^{*}$ & & & & 11,04 \\
\hline g12c9 & 11 & 100 & 190 & 120 & 170 & 100 & 2341,5 & 2342 & 3 & 2355 & 6,98 \\
\hline g12c10 & 10 & 100 & 190 & 70 & 270 & 150 & $2614^{*}$ & & & & 11,81 \\
\hline \hline
\end{tabular}

Table 3: Computational Results 3. 